\title{
Nonlocal energy density functionals for low-energy nuclear structure
}

\author{
F Raimondi ${ }^{1}$, K Bennaceur ${ }^{2,3}$, J Dobaczewski ${ }^{3,4}$ \\ ${ }^{1}$ TRIUMF, 4004 Wesbrook Mall, Vancouver, British Columbia V6T 2A3, Canada \\ ${ }^{2}$ Université de Lyon, F-69003 Lyon, France; Institut de Physique Nucléaire de Lyon, \\ CNRS/IN2P3, Université Lyon 1, F-69622 Villeurbanne Cedex, France \\ ${ }^{3}$ Department of Physics, PO Box 35 (YFL), FI-40014 University of Jyväskylä, \\ Finland \\ ${ }^{4}$ Institute of Theoretical Physics, Faculty of Physics, University of Warsaw, ul. Hoża \\ 69, PL-00681 Warsaw, Poland \\ E-mail: fraimondi@triumf.ca
}

\begin{abstract}
We introduce a finite-range pseudopotential built as an expansion in derivatives up to next-to-next-to-next-to-leading order $\left(\mathrm{N}^{3} \mathrm{LO}\right)$ and we calculate the corresponding nonlocal energy density functional (EDF). The coupling constants of the nonlocal EDF, for both finite nuclei and infinite nuclear matter, are expressed through the parameters of the pseudopotential. All central, spin-orbit, and tensor terms of the pseudopotential are derived both in the spherical-tensor and Cartesian representation. At next-to-leading order (NLO), we also derive relations between the nonlocal EDF expressed in the spherical-tensor and Cartesian formalism. Finally, a simplified version of the finite-range pseudopotential is considered, which generates the EDF identical to that generated by a local potential.
\end{abstract}




\section{Introduction}

Since the discovery of the neutron by Chadwick in 1932, the quest for nuclear interactions has been driving an intense research in nuclear physics. Nuclei are quantum objects composed of correlated components, the nucleons, whose mutual interactions are governed by the low-energy limit of quantum chromodynamics. On the one hand, the contact between the field theory of quarks and gluons and low-energy nuclear structure has been set in the framework of chiral effective field theory [1, 2]. On the other hand, the concept of an effective theory (ET) is powerful enough to embrace a larger set of nuclear structure models, including nuclear potentials employed in phenomenological self-consistent approaches [3].

One of the main features of nuclear forces in the vacuum is the fact that nucleons interact over distances that are comparable with their sizes. Therefore, even when contact or zero-range forces might be adopted for computational reasons, to obtain accurate description of nuclear observables the introduction of finite-range effects is mandatory. In the domain of phenomenological models, different approaches resort to some degree of approximation in describing how nucleons effectively interact in nuclear medium. In mean-field calculations, the use of finite-range interactions was pioneered by Brink and Boecker [4] and extended by Gogny [5, 6], who argued that long and intermediate range of the nuclear in-medium effective force should be included explicitly. Nonetheless, the zero-range approximation was also widely and successfully employed in mean-field calculations, e.g., for determining binding energies and radii, using the zero-range Skyrme interactions [7, 8]. In this case, the finite-range character of the interaction was mimicked through a dependence on relative momenta of the interacting nucleons.

At present, an increased availability of powerful computational resources makes the option of finite-range interaction more and more viable. Furthermore, we tend to regard phenomenological interactions as low-energy in-medium manifestations of the nuclear force, rather than competing and mutually-exclusive approximations of an underlying 'true' potential. This explains the intense ongoing work devoted to improving the form of the phenomenological energy density functionals and interactions [9, 10, 11, 12, 13], along with the recent progress in elaborating new parametrizations of existing ones [14, 15, 16]. The zero-range standard Skyrme interaction has been enriched with higherorder derivative terms [11], whereas extensions to three-body component of the force have been envisaged in both nonlocal [10] and contact [13] forces.

In a recent work [3], we showed that it is possible to apply in a consistent way the ET methodology to low-energy nuclear physics. There we presented a proof-ofprinciple study concerning the convergence, independence of regularization scale, and naturalness of a new class of effective interactions, introduced as regularized zero-range pseudopotentials. In another study [17], we introduced a regularized interaction that was a genuine force, that is, it was density independent, and built as an expansion in relative momenta. This latter interaction has proven to be able to capture the relevant 
physics in the mechanism of saturation but the effective mass. The absence of densitydependent term makes the regularized pseudopotentials suitable for beyond-mean-field calculations, without divergences at high momenta and other pathological behaviors affecting the functionals in multi-reference EDF calculations [18, 19], that is, when standard techniques, like symmetry restoration or Generator Coordinate Method in its most general form, are employed.

In this paper we introduce the general formalism describing the finite-range regularized pseudopotential and nonlocal EDFs obtained by the averaging procedure over the uncorrelated nuclear wavefunction. By considering a finite-range interaction, we get a functional which is bilinear in densities that are built by coupling differential operator to the one-body density matrix. We consider here the expansion in derivatives up the sixth order, corresponding to the $\mathrm{N}^{3} \mathrm{LO}$ functional. Such a functional is genuinely nonlocal, as compared to the quasilocal form of the EDF stemming from the zero-range pseudopotential. The derived EDF is richer in numbers of terms and variety of tensorial couplings of densities to bilinear scalars, as compared to its quasilocal version. This implies, in principle, a functional that has the potential of being more flexible and predictive, but it also implies a larger parameter space to be optimized to nuclear data. The derived regularized pseudopotentials, along with the corresponding nonlocal EDFs, are ready for the optimization procedure, which is the natural follow-up of this formal development, and is now being carried out using the new implementations built within the solver HFODD [20, 21, 22].

The contents of the paper is as follows. In Sec. 2 we present the construction of

the nonlocal EDF from the finite-range pseudopotential. In particular, in Sec. 2.1 we introduce the main features of the finite-range pseudopotential and in Sec. 2.2 we present the nonlocal EDF up to sixth order in derivatives. In Sec. 3 we reduce our results to the case of infinite nuclear matter, whereas in Sec. 4 we compare the NLO EDF derived in the spherical-tensor formalism to that derived in the Cartesian representation. In Sec. 5 we consider a reduced version of the finite-range pseudopotential, for which only the central part of the force is taken into account and a simplified dependence on the relative momenta is assumed. Section 6 presents construction of higher-order spin-orbit (SO) and tensor terms in the Cartesian representation. Finally, our conclusions are presented in Sec. 7

\section{General form of the nonlocal nuclear functional in spherical-tensor formalism}

In this section, we first build the finite-range momentum-dependent two-body pseudopotential, based on general symmetry conditions, and next we derive a nonlocal nuclear EDF by the Hartree-Fock (HF) averaging of the pseudopotential over an uncorrelated many-body wavefunction. In this sense, the pseudopotential can be regarded as an EDF generator, and not as a true in-medium interaction. The derivation from the pseudopotential guarantees the functional to be free from the self-interaction problem. 
Indeed, after the EDF is built, all results are obtained by minimizing the EDF with respect to Kohn-Sham orbitals, whereupon the underlying pseudopotential is never more explicitly invoked. Moreover, the proposed nonlocal EDF is meant to be an approximation of the exact functional whose existence is guaranteed by the variational principle. As such, the corresponding results must be regarded as full solutions and not as pertaining to the HF approximation, which is the first order of the many-body perturbation theory.

\subsection{Finite-range pseudopotential with higher-order momenta}

The pseudopotential that we introduce here can be regarded as a common extension of the most successful nuclear phenomenological interactions: the Skyrme and Gogny forces. Indeed, the central part of our pseudopotential is finite-range and momentumdependent, whereas the one of the Skyrme force is momentum-dependent but zero-range and the one of the Gogny force is finite-range but momentum-independent. Similarly as for the Skyrme force, we build the pseudopotential in terms of an expansion in relative momenta, and similarly as for the Gogny force, we use finite-range formfactors. We note here that the constructed pseudopotential has only one finite-range parameter, allowing us to interpret that parameter as a regularization scale, according to the ET philosophy [3].

Following closely the methodology of introducing higher-order derivatives of zero-range pseudopotentials [11, we obtain the general form of the finite-range pseudopotential by coupling derivative operators with spin operators in the sphericaltensor formalism. The complete list of the building blocks of the tensors can be found in Ref. 9]. We obtain the finite-range version by simply substituting the zero-range Dirac delta with a regularized (smeared) delta,

$$
g_{a}(\boldsymbol{r})=g_{a}(r)=\frac{e^{-\frac{r^{2}}{a^{2}}}}{(a \sqrt{\pi})^{3}},
$$

where $r=|\boldsymbol{r}|=\left|\boldsymbol{r}_{2}-\boldsymbol{r}_{1}\right|$ and $\boldsymbol{r}_{2}$ and $\boldsymbol{r}_{1}$ are positions of the two interacting particles.

In such a formalism, the general expression for the antisymmetrized finite-range pseudopotential reads,

$$
\hat{V}=\sum_{\substack{\tilde{n}^{\prime} \tilde{L}^{\prime}, \tilde{t} \\ \tilde{n} \tilde{L}, v_{12} \tilde{S}}} C_{\tilde{n} \tilde{L}, v_{12} \tilde{S}}^{\tilde{n}^{\prime} \tilde{L}^{\prime}, \tilde{t}} \hat{V}_{\tilde{n} \tilde{L}, v_{12} \tilde{S}}^{\tilde{n}^{\prime} \tilde{L}^{\prime}, \tilde{t}},
$$

where each term, which is built according to the symmetries discussed in Ref. [1] and multiplied by the corresponding strength parameter $C_{\tilde{n} \tilde{L}, v_{12} \tilde{S}}^{\tilde{n}^{\prime} \tilde{L}^{\prime}, \tilde{t}}$, is given by

$$
\begin{aligned}
\hat{V}_{\tilde{n} \tilde{L}, v_{12} \tilde{S}}^{\tilde{n}^{\prime} \tilde{L}^{\prime}, \tilde{t}}= & \frac{1}{2} i^{v_{12}}\left(\left[\left[K_{\tilde{n}^{\prime} \tilde{L}^{\prime}}^{\prime} K_{\tilde{n} \tilde{L}}\right]_{\tilde{S}} \hat{S}_{v_{12} \tilde{S}}\right]_{0}\right. \\
& \left.+(-1)^{v_{12}+\tilde{S}}\left[\left[K_{\tilde{n} \tilde{L}}^{\prime} K_{\tilde{n}^{\prime} \tilde{L}^{\prime}}\right]_{\tilde{S}} \hat{S}_{v_{12} \tilde{S}}\right]_{0}\right)\left(\hat{P}^{\tau}\right)^{\tilde{t}} \\
& \times\left(1-\hat{P}^{M} \hat{P}^{\sigma} \hat{P}^{\tau}\right) \delta\left(\boldsymbol{r}_{1}^{\prime}-\boldsymbol{r}_{1}\right) \delta\left(\boldsymbol{r}_{2}^{\prime}-\boldsymbol{r}_{2}\right) g_{a}(r) .
\end{aligned}
$$


For the sake of completeness, we recall here definitions of tensors that appear in Eq. (3). Each term of the pseudopotential is a scalar with a composite internal structure and is built of higher-rank spherical tensors in the space, spin, and isospin coordinates. Higher-order tensor derivatives $K_{\tilde{n} \tilde{L}}$ are built of relative momenta $\boldsymbol{k}=\left(\boldsymbol{\nabla}_{1}-\boldsymbol{\nabla}_{2}\right) / 2 i$, with spherical components

$$
k_{1, \mu=\{-1,0,1\}}=-i\left\{\frac{1}{\sqrt{2}}\left(k_{x}-i k_{y}\right), k_{z}, \frac{-1}{\sqrt{2}}\left(k_{x}+i k_{y}\right)\right\}
$$

that are coupled to $\operatorname{rank} \tilde{L}$, where symbol $\tilde{n}$ denotes the order of the tensor, that is, the number of operators $\boldsymbol{k}$. Operators acting on the primed coordinates, $K_{\tilde{n} \tilde{L}}^{\prime}$, are built in the same way of relative momenta $\boldsymbol{k}^{\prime}=\left(\boldsymbol{\nabla}_{1}^{\prime}-\boldsymbol{\nabla}_{2}^{\prime}\right) / 2 i$.

In the spin space, for particles numbered by $i=1$ or 2 , we have as building blocks the spin unity matrix of rank 0 , denoted as $\sigma_{00}^{(i)}$, and the Pauli matrices $\sigma_{x, y, z}^{(i)}$, which define the spin spherical tensor of rank 1 ,

$$
\sigma_{1, \mu=\{-1,0,1\}}^{(i)}=-i\left\{\frac{1}{\sqrt{2}}\left(\sigma_{x}^{(i)}-i \sigma_{y}^{(i)}\right), \sigma_{z}^{(i)}, \frac{-1}{\sqrt{2}}\left(\sigma_{x}^{(i)}+i \sigma_{y}^{(i)}\right)\right\} .
$$

From these definitions, we can construct tensors of rank up to 2, according to the following symmetrized expression

$$
\hat{S}_{v_{12} \tilde{S}}=\left(1-\frac{1}{2} \delta_{v_{1}, v_{2}}\right)\left(\left[\sigma_{v_{1}}^{(1)} \sigma_{v_{2}}^{(2)}\right]_{\tilde{S}}+\left[\sigma_{v_{2}}^{(1)} \sigma_{v_{1}}^{(2)}\right]_{\tilde{S}}\right)
$$

where $v_{12}=v_{1}+v_{2}$ and $\sigma_{v}^{(i)}$ are the spherical-tensor rank- $v$ Pauli matrices.

The locality delta functions $\delta\left(\boldsymbol{r}_{1}^{\prime}-\boldsymbol{r}_{1}\right) \delta\left(\boldsymbol{r}_{2}^{\prime}-\boldsymbol{r}_{2}\right)$ together with the regularized delta $g_{a}(r)$ must be understood as distributions, whereupon the derivatives in $K_{\tilde{n} \tilde{L}}$ and $K_{\tilde{n} \tilde{L}}^{\prime}$ act. The locality deltas ensure the locality of the energy density only in the zero-range case, whereas it is in principle nonlocal due to the presence of the so called Majorana operator $\hat{P}^{M}$ in the exchange part of the pseudopotential.

In Eq. (3), index $\tilde{t}$, taking either value 0 or 1 , defines the dependence of the pseudopotential on the isospin. This dependence is in addition to that stemming from the exchange term, and is a new element as compared to the case of the zero-range pseudopotential [11].

In principle, one could write down an equivalent version of the antisymmetrized finite-range pseudopotential, where the new dependence on the isospin exchange operator would have been substituted by the Majorana operator exchanging space coordinates, $\hat{P}^{M}$. This would be possible in virtue of the relation $\hat{P}^{\tau} \equiv-\hat{P}^{\sigma} \hat{P}^{M}$, valid when acting on a fermionic wave function. However, the presence of the Majorana operator in the pseudopotential would break the correspondence between the exchange part of the antisymmetrized pseudopotential and the nonlocal sector of the EDF, according to the common understanding of the nonlocal part of the EDF as resulting from the exchange part of the antisymmetrized interaction. While in a zero-range pseudopotential this operator reduces to a phase depending on the order of the relativemomenta tensors [23, 11], in a finite-range interaction this operator acts explicitly, and switches the space coordinates of the two interacting nucleons. Therefore the finiterange character of the interaction, complemented with the action of the Majorana 
operator, would have given rise to the nonlocal form of the functional stemming from the direct interaction. Although such a construction would be perfectly correct and exactly equivalent to that presented below, it would also be counterintuitive, and thus in our study it is not further pursued.

The constructed pseudopotential possesses all symmetries of the nuclear interaction. Referring the reader to Ref. [11] for a comprehensive discussion, here we only list these symmetries, which are rotational symmetry, time-reversal, parity, and Galilean invariance, supplemented with the hermiticity of the pseudopotential operator and indistinguishability principle operating as invariance under exchange of particles 1 and 2 . The pseudopotential (2) is indeed invariant under all the transformations corresponding to the symmetries listed above. This guarantees that the resulting EDF is also invariant with respect to the same symmetries.

All terms of the pseudopotential, derived at zero, second, fourth, and sixth order, $\tilde{n}+\tilde{n}^{\prime}=0,2,4$, and 6 , respectively, are collected in the supplemental material. The supplemental material contains all detailed results of the present study. They were derived by means of symbolic programing, and are presented in readable format as well as in the form directly usable in computer programming. In Table 1 we show numbers of different terms of pseudopotential (2), where we also distinguish between central $(\tilde{S}=0)$, SO $(\tilde{S}=1)$, and tensor $(\tilde{S}=2)$ terms, corresponding to different ranks in the coupling of the relative momenta tensors $K_{\tilde{n} \tilde{L}}$ with the spin operator $\hat{S}_{v_{12} \tilde{S}}$. At each order, the overall numbers of terms equal 4, 14, 30, and 52, giving the total number of 100 terms up to $\mathrm{N}^{3} \mathrm{LO}$. At each order, these numbers are twice larger than the corresponding numbers for the zero-range pseudopotential, which reflects the addition of the new quantum number $\tilde{t}$.

We note, that here we classified tensor terms as those corresponding to $\tilde{S}=2$; however, in the pseudopotential (3) there also appear terms that have purely spacetensor character, e.g., those for $\tilde{S}=0$ and $\tilde{L}=\tilde{L}^{\prime}=2$. However, we know from the generalized Cayley-Hamilton (GCH) theorem [24], that any scalar function of two vectors $\boldsymbol{k}$ and $\boldsymbol{k}^{\prime}$ must have form of a function of three elementary scalars $\boldsymbol{k}^{2}, \boldsymbol{k}^{\prime 2}$, and

$\boldsymbol{k}^{\prime} \cdot \boldsymbol{k}$. Therefore, the central term $(\tilde{S}=0)$ can always be recoupled to the form where terms with $\tilde{L}, \tilde{L}^{\prime}>1$ do not appear. An explicit construction of such a form is presented in the Cartesian coordinates in Sec. 4.

\subsection{Nonlocal nuclear EDF}

The interaction energy, which is the potential part of the EDF, is derived by averaging the pseudopotential $\hat{V}$ (2) over the uncorrelated nuclear wave function expressed as Slater determinant. The functional obtained in this way is in general nonlocal, meaning that it contains terms depending on one-body densities non-diagonal with respect to the spatial coordinates.

If we leave out the exchange operator from the pseudopotential (2), we can define 
Table 1. Numbers of terms of pseudopotential (2) at different orders up to $\mathrm{N}^{3} \mathrm{LO}$. In the second, third, and fourth column, numbers of central $(\tilde{S}=0)$, SO $(\tilde{S}=1)$, and tensor $(\tilde{S}=2)$ terms, respectively, are displayed.

\begin{tabular}{lllll}
\hline Order & $\tilde{S}=0$ & $\tilde{S}=1$ & $\tilde{S}=2$ & Total \\
\hline 0 & 4 & 0 & 0 & 4 \\
2 & 8 & 2 & 4 & 14 \\
4 & 16 & 4 & 10 & 30 \\
6 & 24 & 8 & 20 & 52 \\
$\mathrm{~N}^{3} \mathrm{LO}$ & 52 & 14 & 34 & 100 \\
\hline
\end{tabular}

it as $\hat{V}=\mathcal{V}\left(1-\hat{P}^{M} \hat{P}^{\sigma} \hat{P}^{\tau}\right)$. This allows us to express the EDF in the following way,

$$
\begin{aligned}
\mathcal{E}= & \frac{1}{2} \int \mathrm{d} \boldsymbol{r}_{1}^{\prime} \mathrm{d} \boldsymbol{r}_{2}^{\prime} \mathrm{d} \boldsymbol{r}_{1} \mathrm{~d} \boldsymbol{r}_{2} \sum_{\substack{\sigma_{1} \sigma_{2} \\
\sigma_{1}^{\prime} \sigma_{2}^{\prime}}} \sum_{\substack{\tau_{1}^{\prime} \tau_{2} \\
\tau_{2}^{\prime}}} \mathcal{V}\left(\boldsymbol{r}_{1}^{\prime} \sigma_{1}^{\prime} \tau_{1}^{\prime} \boldsymbol{r}_{2}^{\prime} \sigma_{2}^{\prime} \tau_{2}^{\prime}, \boldsymbol{r}_{1} \sigma_{1} \tau_{1} \boldsymbol{r}_{2} \sigma_{2} \tau_{2}\right) \\
& \left(\rho\left(\boldsymbol{r}_{1} \sigma_{1} \tau_{1}, \boldsymbol{r}_{1}^{\prime} \sigma_{1}^{\prime} \tau_{1}^{\prime}\right) \rho\left(\boldsymbol{r}_{2} \sigma_{2} \tau_{2}, \boldsymbol{r}_{2}^{\prime} \sigma_{2}^{\prime} \tau_{2}^{\prime}\right)-\rho\left(\boldsymbol{r}_{2} \sigma_{2} \tau_{2}, \boldsymbol{r}_{1}^{\prime} \sigma_{1}^{\prime} \tau_{1}^{\prime}\right) \rho\left(\boldsymbol{r}_{1} \sigma_{1} \tau_{1}, \boldsymbol{r}_{2}^{\prime} \sigma_{2}^{\prime} \tau_{2}^{\prime}\right)\right),
\end{aligned}
$$

where the two-body spin-isospin (non-antisymmetrized) matrix elements of $\mathcal{V}$ are defined as

$$
\mathcal{V}\left(\boldsymbol{r}_{1}^{\prime} s_{1}^{\prime} t_{1}^{\prime} \boldsymbol{r}_{2}^{\prime} s_{2}^{\prime} t_{2}^{\prime}, \boldsymbol{r}_{1} s_{1} t_{1} \boldsymbol{r}_{2} s_{2} t_{2}\right)=\left\langle s_{1}^{\prime} t_{1}^{\prime}, s_{2}^{\prime} t_{2}^{\prime}\left|\mathcal{V}\left(\boldsymbol{r}_{1}^{\prime}, \boldsymbol{r}_{2}^{\prime}, \boldsymbol{r}_{1}, \boldsymbol{r}_{2}\right)\right| s_{1} t_{1}, s_{2} t_{2}\right\rangle,
$$

and $\rho\left(\boldsymbol{r}_{1} s_{1} t_{1}, \boldsymbol{r}_{1}^{\prime} s_{1}^{\prime} t_{1}^{\prime}\right)$ and $\rho\left(\boldsymbol{r}_{2} s_{2} t_{2}, \boldsymbol{r}_{2}^{\prime} s_{2}^{\prime} t_{2}^{\prime}\right)$, are the one-body density matrices in spinisospin channels. In Eq. (7), the two terms that are bilinear in densities lead to the standard direct and exchange terms, which are respectively, local and nonlocal in space coordinates.

We performed derivations of average energies (7) separately for all terms of the pseudopotential (2). The final result of this derivation is given by linear combinations of terms of the EDF appearing on the rhs of the following expression,

$$
\left\langle C_{\tilde{n} \tilde{L}, v_{12} \tilde{S}}^{\tilde{n}^{\prime} \tilde{L}^{\prime}, \tilde{t}} \hat{V}_{\tilde{n} \tilde{L}, v_{12} \tilde{S}}^{\tilde{n}^{\prime} \tilde{L}^{\prime}, \tilde{t}}\right\rangle=\sum C_{a, \alpha, Q}^{a^{\prime}, \alpha^{\prime}, t, \mathcal{L}} T_{a, \alpha, Q}^{a^{\prime}, \alpha^{\prime}, t, \mathcal{L}} .
$$

In this expression, $C_{a, \alpha, Q}^{a^{\prime}, \alpha^{\prime}, t, \mathcal{L}}$ and $T_{a, \alpha, Q}^{a^{\prime}, \alpha^{\prime}, t, \mathcal{L}}$ denote, respectively, the coupling constants and terms of the EDF according to the compact notation introduced in Ref. [25], where the Greek indices $\alpha=\left\{n_{\alpha} S_{\alpha} v_{\alpha} J_{\alpha}\right\}$ and Roman indices $a=\left\{m_{a} I_{a}\right\}$ combine all the quantum numbers of the local densities $\rho_{\alpha}(\boldsymbol{r})$ and derivative operators $D_{a}$, as defined below. The spherical-tensor formalism for the higher-order EDF has been developed in Ref. [9]. Because here we treat the isospin degree of freedom explicitly, and because we deal with a nonlocal functional, we enriched the notation by adding superscripts $t$, which denote the isoscalar $(t=0)$ or isovector $(t=1)$ channels, and by adding labels $\mathcal{L}$ that distinguish between local $(\mathcal{L}=L)$ and nonlocal $(\mathcal{L}=N)$ terms of the functional. Index $Q$ corresponds to the total rank of densities, which are coupled to a scalar.

The formalism developed in Ref. [9], along with the straightforward extension to the isospace introduced in Ref. [12], which originally pertained to quasilocal higher-order EDF and zero-range pseudopotential, can easily be accommodated to express the EDF 
discussed in this paper. Then, direct (local) terms of the functional, bilinear in local densities, read

$$
T_{a, \alpha, Q}^{a^{\prime}, \alpha^{\prime}, t, L}=\int \mathrm{d} \boldsymbol{r}_{1} \mathrm{~d} \boldsymbol{r}_{2} g_{a}(\boldsymbol{r})\left[\left[\left[D_{a^{\prime}} \rho_{\alpha^{\prime}}^{t}\left(\boldsymbol{r}_{1}\right)\right]_{Q}\left[D_{a} \rho_{\alpha}^{t}\left(\boldsymbol{r}_{2}\right)\right]_{Q}\right]^{0}\right]_{0} .
$$

They have been obtained using the integration by parts to transfer all derivatives onto the density matrices, and then employing the locality deltas to perform integrations over two out of four space coordinates. In Eq. (10), subscripts and superscripts denote the standard coupling of the angular momentum and isospin, respectively, and the higherorder derivative operators $D_{a}$ of order $m_{a}$ and rank $I_{a}$, are built from the order-one, rank-one derivative operators,

$$
D_{11}=2 \nabla_{1} \quad \text { or } \quad D_{11}=2 \nabla_{2}
$$

depending on whether they act on variables $\boldsymbol{r}_{1}$ or $\boldsymbol{r}_{2}$.

In an analogous way, exchange (nonlocal) terms of the functional read

$$
T_{a, \alpha, Q}^{a^{\prime}, \alpha^{\prime}, t, N}=\int \mathrm{d} \boldsymbol{r}_{1} \mathrm{~d} \boldsymbol{r}_{2} g_{a}(\boldsymbol{r})\left[\left[\left[D_{a^{\prime}} \rho_{\alpha^{\prime}}^{t}\left(\boldsymbol{r}_{1}, \boldsymbol{r}_{2}\right)\right]_{Q}\left[D_{a} \rho_{\alpha}^{t}\left(\boldsymbol{r}_{2}, \boldsymbol{r}_{1}\right)\right]_{Q}\right]^{0}\right]_{0},
$$

where the nonlocal densities are defined as,

$$
\rho_{\alpha}^{t}\left(\boldsymbol{r}_{1}, \boldsymbol{r}_{2}\right)=\left[K_{n S} \rho_{v}^{t}\left(\boldsymbol{r}_{1}, \boldsymbol{r}_{2}\right)\right]_{J},
$$

with the order- $n$ and rank- $S$ relative derivative operators $K_{n S}$ acting on nonlocal densities and built from the relative-momentum operators,

$$
K_{11}=\frac{1}{2 \mathrm{i}}\left(\nabla_{1}-\nabla_{2}\right)
$$

Derivative operators $D_{a}$ of order $m_{a}$ and rank $I_{a}$, are built from the building blocks,

$$
D_{11}=\nabla_{1}+\nabla_{2}
$$

We do not use different notations for operators (11) and (15) - which one of them is used is clear from the context.

The coupling constants of the functional that we consider in the present study do not depend on densities. Therefore, in principle, in expressions (10) and (12) one could perform integrations by parts and thus, in an attempt to achieve the same form as that for the quasilocal EDF considered in Ref. [9], transfer the derivative operators $D_{a^{\prime}}$ onto the second density. However, this transformation would have created a series of extra terms produced by the action of the derivative operators onto the regularized delta, in such a way that the functional would have had a mixed form composed of derivatives of densities and an expansion in the parameter $a$ of (1). For this reason, we keep the form of Eqs. (10) and (12) with the derivatives operators acting on both densities.

Explicit calculations of linear combinations in Eq. (9) are formally identical to those performed for the zero-range pseudopotential (see Sec. III of Ref. [11] for details). In this Section, we present general results for the finite-range pseudopotential (2), which we also supplement by those pertaining to the symmetric spin-saturated nuclear matter. Table 2 
Table 2. Numbers of terms defined in Eqs. (10) and (12) of different orders in the EDF up to $\mathrm{N}^{3} \mathrm{LO}$, given for one isospin channel. In the second, third, and fourth columns, numbers terms stemming from central $(\tilde{S}=0)$, SO $(\tilde{S}=1)$, and tensor $(\tilde{S}=2)$ finite-range pseudopotential are given, respectively. The last column gives the numbers of terms of the EDF when it is applied to the symmetric spin-saturated nuclear matter.

\begin{tabular}{llllll}
\hline Order & $\tilde{S}=0$ & $\tilde{S}=1$ & $\tilde{S}=2$ & Total & Nuclear matter \\
\hline 0 & 4 & 0 & 0 & 4 & 2 \\
2 & 24 & 8 & 16 & 36 & 3 \\
4 & 144 & 64 & 114 & 222 & 8 \\
6 & 640 & 336 & 564 & 1010 & 12 \\
$\mathrm{~N}^{3} \mathrm{LO}$ & 776 & 408 & 694 & 1272 & 25 \\
\hline
\end{tabular}

lists the numbers of independent terms (10) and (12) of the functional obtained from the finite-range pseudopotential. Each allowed combination of indexes $\left(a^{\prime}, \alpha^{\prime}, t, a, \alpha, Q\right)$ gives a pair of EDF terms, one local and another one nonlocal; therefore, the numbers shown are twice the numbers of such allowed combinations. The fact that the isospin does not couple with operators belonging to spin and position-coordinate space, along with the requirement that the EDF is isoscalar, implies that isoscalar and isovector densities, respectively $t=0$ and $t=1$, give rise to two isospin channels in the functional having the same structure. Therefore only one isospin channel is accounted for in table 2 , For the symmetric nuclear matter, the isovector terms do not contribute.

Table 2 also displays numbers of EDF independent terms obtained separately from the central $(\tilde{S}=0)$, $S O(\tilde{S}=1)$, and tensor $(\tilde{S}=2)$ terms of the finite-range pseudopotential. Strictly speaking, the EDF cannot be divided into central and tensor contributions, even though the SO part of the EDF is decoupled from the other two. This is at the origin of the functional terms that mix scalar and vector densities in spin spaces. However there are terms of the EDF that can be produced by both central and tensor terms of the pseudopotential. This explains why the sums of terms in the second, third, and fourth columns do not equal to the corresponding values in the fifth column.

2.2.1. Zero-order (LO) EDF. In order to illustrate how the nonlocal EDF derives from a finite-range pseudopotential, we show explicitly the functional obtained from the averaging of the zero-order finite-range pseudopotential $\hat{V}^{(0)}$ over the nuclear Slater determinant. Below, symbols $\hat{V}^{(n)}$ and $\mathcal{E}^{(n)}$ denote, respectively, the order- $n$ regularized pseudopotential and functional.

The four terms of the pseudopotential at zero order are,

$$
\hat{V}^{(0)}=C_{00,00}^{00,0} \hat{V}_{00,00}^{00,0}+C_{00,00}^{00,1} \hat{V}_{00,00}^{00,1}+C_{00,20}^{00,0} \hat{V}_{00,20}^{00,0}+C_{00,20}^{00,1} \hat{V}_{00,20}^{00,1} .
$$

The complete expression for the zero-order nonlocal EDF obtained from (16) reads

$$
\mathcal{E}^{(0)}=\left(\frac{C_{00,00}^{00,0}}{2}+\frac{C_{00,00}^{00,1}}{4}\right) T_{00,000,0}^{00,000,0, L}
$$




$$
\begin{aligned}
& +\left(-\frac{C_{00,20}^{00,0}}{2}-\frac{C_{00,20}^{00,1}}{4}\right) T_{00,0011,1}^{00,0011, L} \\
& +\left(\frac{\sqrt{3}}{4} C_{00,00}^{00,1}\right) T_{00,0000,0}^{00,0000,1, L} \\
& +\left(-\frac{\sqrt{3}}{4} C_{00,20}^{00,1}\right) T_{00,0011,1}^{00,001,1, L} \\
& +\left(-\frac{\left.C_{00,00}^{00,0}-\frac{C_{00,00}^{00,1}}{8}+\frac{\sqrt{3}}{8} C_{00,20}^{00,0}+\frac{\sqrt{3}}{4} C_{00,20}^{00,1}\right) T_{00,000,0}^{00,000, N}}{8}\right) T_{00,0011,1}^{00,011,0} \\
& +\left(-\frac{\sqrt{3}}{8} C_{00,00}^{00,0}-\frac{\sqrt{3}}{4} C_{00,00}^{00,1}-\frac{C_{00,20}^{00,0}}{8}-\frac{C_{00,20}^{00,1}}{4}\right) \\
& +\left(-\frac{\sqrt{3}}{8} C_{00,00}^{00,0}+\frac{3}{8} C_{00,20}^{00,0}\right) T_{00,0000,0}^{00,0000,1, N} \\
& +\left(-\frac{3}{8} C_{00,00}^{00,0}-\frac{\sqrt{3}}{8} C_{00,20}^{00,0}\right) T_{00,0011,1}^{00,0011,1, N} .
\end{aligned}
$$

In Eq. (17), we have eight zero-order, 4 local and 4 nonlocal, or 4 isoscalar and 4 isovector. coupling constants expressed explicitly as linear combinations of the four zero-order pseudopotential parameters given in Eq. (16).

2.2.2. Beyond the $L O E D F$. The 72 second-order isoscalar and isovector coupling constants of the nonlocal EDF, expressed by the 14 second-order finite-range pseudopotential parameters, are collected in the supplemental material according to the following formula using the grouped index defined as $\left(a, \alpha, Q, a^{\prime}, \alpha^{\prime}, t, \mathcal{L}\right) \equiv \mathcal{A}$,

$$
\begin{aligned}
C_{a, \alpha, Q}^{a^{\prime}, \alpha^{\prime}, t, \mathcal{L}}= & \sum_{\tilde{t}=0}^{1} a_{\mathcal{A}, \tilde{t}} C_{00,00}^{20, \tilde{t}}+b_{\mathcal{A}, \tilde{t}} C_{00,20}^{20, \tilde{t}}+c_{\mathcal{A}, \tilde{t}} C_{00,22}^{22, \tilde{t}}+d_{\mathcal{A}, \tilde{t}} C_{11,00}^{11, \tilde{t}}+e_{\mathcal{A}, \tilde{t}} C_{11,11}^{11, \tilde{t}} \\
& +f_{\mathcal{A}, \tilde{t}} C_{11,20}^{11, \tilde{t}}+g_{\mathcal{A}, \tilde{t}} C_{11,22}^{11, \tilde{t}} .
\end{aligned}
$$

In Eq. (18), and in Eqs. (19) and (20) below, numerical coefficients $a_{\mathcal{A}, \tilde{t}}, b_{\mathcal{A}, \tilde{t}}, \ldots$, express the EDF coupling constants as linear combinations of the finite-range pseudopotential parameters.

Similar expressions for the fourth-order (sixth-order) 444 (2020) coupling constants that are related to $30(52)$ parameters of the finite-range pseudopotential can also be found in the supplemental material, according to the formulas

$$
\begin{aligned}
C_{a, \alpha, Q}^{a^{\prime}, \alpha^{\prime}, t, \mathcal{L}}= & \sum_{\tilde{t}=0}^{1} a_{\mathcal{A}, \tilde{t}} C_{00,00}^{40, \tilde{t}}+b_{\mathcal{A}, \tilde{t}} C_{00,20}^{40, \tilde{t}}+c_{\mathcal{A}, \tilde{t}} C_{00,22}^{42, \tilde{t}}+d_{\mathcal{A}, \tilde{t}} C_{11,00}^{31, \tilde{t}}+e_{\mathcal{A}, \tilde{t}} C_{11,11}^{31, \tilde{t}} \\
& +f_{\mathcal{A}, \tilde{t}} C_{11,20}^{31, \tilde{t}}+g_{\mathcal{A}, \tilde{t}} C_{11,22}^{31, \tilde{t}}+h_{\mathcal{A}, \tilde{t}} C_{11,22}^{33, \tilde{t}}+i_{\mathcal{A}, \tilde{t}} C_{20,00}^{20, \tilde{t}}+j_{\mathcal{A}, \tilde{t}} C_{20,20}^{20, \tilde{t}} \\
& \left.+k_{\mathcal{A}, \tilde{t}} C_{20,22}^{22, \tilde{t}}+l_{\mathcal{A}, \tilde{t}} C_{22,00}^{22, \tilde{t}}+m_{\mathcal{A}, \tilde{t}} C_{22,11}^{22, \tilde{t}}+n_{\mathcal{A}, \tilde{t}} C_{22,20}^{22, \tilde{t}}+o_{\mathcal{A}, \tilde{t}} C_{22, \underline{t} 29}^{22, \tilde{t}} 9\right)
\end{aligned}
$$


and

$$
\begin{aligned}
C_{a, \alpha, Q}^{a^{\prime}, \alpha^{\prime}, t, \mathcal{L}}= & \sum_{\tilde{t}=0}^{1} a_{\mathcal{A}, \tilde{t}} C_{00,00}^{60, \tilde{t}}+b_{\mathcal{A}, \tilde{t}} C_{00,20}^{60, \tilde{t}}+c_{\mathcal{A}, \tilde{t}} C_{00,22}^{62, \tilde{t}}+d_{\mathcal{A}, \tilde{t}} C_{11,00}^{51, \tilde{t}}+e_{\mathcal{A}, \tilde{t}} C_{11,11}^{51, \tilde{t}} \\
& +f_{\mathcal{A}, \tilde{t}} C_{11,20}^{31, \tilde{t}}+g_{\mathcal{A}, \tilde{t}} C_{11,22}^{51, \tilde{t}}+h_{\mathcal{A}, \tilde{t}} C_{11,22}^{53, \tilde{t}}+i_{\mathcal{A}, \tilde{t}} C_{20,00}^{40, \tilde{t}}+j_{\mathcal{A}, \tilde{t}} C_{20,20}^{40, \tilde{t}} \\
& +k_{\mathcal{A}, \tilde{t}} C_{20,22}^{42, \tilde{t}}+l_{\mathcal{A}, \tilde{t}} C_{22,00}^{42, \tilde{t}}+m_{\mathcal{A}, \tilde{t}} C_{22,11}^{42, \tilde{t}}+n_{\mathcal{A}, \tilde{t}} C_{22,20}^{42, \tilde{t}}+o_{\mathcal{A}, \tilde{t}} C_{22,22}^{40, \tilde{t}} \\
& +p_{\mathcal{A}, \tilde{t}} C_{22,22}^{42, \tilde{t}}+q_{\mathcal{A}, \tilde{t}} C_{22,22}^{44, \tilde{t}}+r_{\mathcal{A}, \tilde{t}} C_{31,00}^{31, \tilde{t}}+s_{\mathcal{A}, \tilde{t}} C_{31,11}^{31, \tilde{t}}+u_{\mathcal{A}, \tilde{t}} C_{31,20}^{31, \tilde{t}} \\
& +v_{\mathcal{A}, \tilde{t}} C_{31,22}^{31, \tilde{t}}+w_{\mathcal{A}, \tilde{t}} C_{31,22}^{33, \tilde{t}}+x_{\mathcal{A}, \tilde{t}} C_{33,00}^{33, \tilde{t}}+y_{\mathcal{A}, \tilde{t}} C_{33,11}^{33, \tilde{t}}+z_{\mathcal{A}, \tilde{t}} C_{33,20}^{33, \tilde{t}} \\
& +\beta_{\mathcal{A}, \tilde{t}} C_{33,22}^{33, \tilde{t}},
\end{aligned}
$$

respectively.

Besides the coupling constants expressed through the linear combinations in Eqs. (19) and (20) there are 6 (38) more coupling constants for each isospin channel at fourth-order (sixth-order), corresponding to EDF terms that are allowed by the general symmetries of the functional, but that are forced to be equal to zero once the functional is derived from the pseudopotential. Therefore, the number of terms of the fourthorder (sixth-order) EDF when not derived from the pseudopotential is 228 (1048) in each isospin channel. The complete lists of these fourth-order and sixth-order vanishing coupling constants are included in the supplemental material. Indeed as we verified for the quasilocal EDF obtained from the zero-range pseudopotential, also for the finiterange one, the explicit Galilean invariance of the pseudopotential (3) yields the Galilean invariance of the derived nonlocal functional. The coupling constants of the terms which are not Galilean invariant turn out to be equal to zero when the derivation of the functional from the pseudopotential is performed. Moreover using expressions (18)(20), one can, in principle, determine constraints between the nonvanishing coupling constants of the EDF that enforce Galilean symmetry. For example, at NLO this task can be realized by inverting two subsets of 14 linear combinations (18), chosen arbitrarily within each local and nonlocal part separately. The only requirement is in selecting linear combinations that lead to a nonsingular matrix. In this way, one can express the remaining coupling constants through the 14 selected as independent ones. The two sets of linear combinations, one for the local component and one for the nonlocal component of the EDF, form together the constraints on the functional obtained by imposing the Galilean invariance.

These linear combinations form subsets of equivalent relations in each isospin space. This is owing to the specularity of the EDF with respect to the isospin. In the local and nonlocal sectors of the functionals, this is owing to the fact that linear combinations between EDF terms can be established only for terms with the same kind of densities, local or nonlocal.

Then, considering at NLO both isospin channels $(t=0,1)$ and both local and nonlocal sectors of the functional $(\mathcal{L}=L, N), 44$ dependent coupling constants are 
equal to specific linear combinations of the 28 ones, that is,

$$
\begin{aligned}
& C_{00,1110,0}^{00,1110, t, \mathcal{L}}=-\frac{C_{00,2011,1}^{00,0011, t, \mathcal{L}}}{3}-\frac{\sqrt{5} C_{00,2211,1}^{00,0011, t, \mathcal{L}}}{3}, \\
& C_{00,1111,1}^{00,1111, t \mathcal{L}}=-\frac{C_{00,2011,1}^{00,0011, t, \mathcal{L}}}{\sqrt{3}}+\frac{1}{2} \sqrt{\frac{5}{3}} C_{00,2211,1}^{00,0011, t, \mathcal{L}}, \\
& C_{00,1112,2}^{00,1112, t, \mathcal{L}}=-\frac{\sqrt{5} C_{00,2011,1}^{00,0011, t, \mathcal{L}}}{3}-\frac{C_{00,2211,1}^{00,0011, t, \mathcal{L}}}{6}, \\
& C_{00,2000,0}^{00,0000, t, \mathcal{L}}=-C_{00,1101,1}^{00,1101, \mathcal{L}} \\
& C_{11,0000,1}^{00,1111, t, \mathcal{L}}=-C_{11,1101,1}^{00,0011, t, \mathcal{L}}, \\
& C_{11,0011,1}^{00,1101, \mathcal{L}}=C_{11,1101,1}^{00,0011, \mathcal{L}} \\
& C_{11,0011,1}^{11,0011, t, \mathcal{L}}=-\frac{2 C_{11,0011,0}^{11,0011, t, \mathcal{L}}}{\sqrt{3}}+\sqrt{\frac{5}{3}} C_{11,0011,2}^{11,0011, t, \mathcal{L}}, \\
& C_{11,1111,0}^{00,0000, \mathcal{L}}=C_{11,1101,1}^{00,0011, \mathcal{L}}, \\
& C_{20,0000,0}^{00,000, t, \mathcal{L}}=-C_{11,0000,1}^{11,0000, \mathcal{L}}, \\
& C_{20,0011,1}^{00,0011, t, \mathcal{L}}=\frac{C_{11,0011,0}^{11,0011, \mathcal{L}}}{3}-\frac{2 \sqrt{5} C_{11,0011,2}^{11,0011, t, \mathcal{L}}}{3}, \\
& C_{22,0011,1}^{00,0011, t, \mathcal{L}}=-\frac{2 \sqrt{5} C_{11,0011,0}^{11,0011, t, \mathcal{L}}}{3}+\frac{2 C_{11,0011,2}^{11,0011, t, \mathcal{L}}}{3} .
\end{aligned}
$$

Equations (21) $-(25)$ are formally equivalent to the constraints found for the same symmetry in the case of quasilocal EDF [9]. This is so, because the corresponding

terms have exactly the same tensor forms as those found in the functional stemming from the zero-range pseudopotential. The remaining linear combinations (26)-(31) have no analogs in the quasilocal EDF, because they involve terms that are absent in the quasilocal version of the functional. We also note that there are no terms corresponding to unrestricted coupling constants; indeed, the Galilean symmetry forces all the nonvanishing coupling constants to enter in specific linear combinations.

\section{Nonlocal EDF for the symmetric spin-saturated nuclear matter}

For the symmetric spin-saturated homogeneous nuclear matter, the EDF coupling constants expressed in terms of the pseudopotential parameters can easily be obtained from those derived for finite nuclei. Indeed, in this former system, isovector densities are zero because of the equality of proton and neutron densities, gradients of local densities are zero because of its homogeneity, and all tensors of rank greater than zero vanish because of its isotropy.

At zero-order, expressions relating the EDF non-vanishing coupling constants to pseudopotential parameters can be read from the first and fifth lines of Eq. (17). They explicitly read,

$$
C_{00,0000,0}^{00,0000,0, L}=\frac{C_{00,00}^{00,0}}{2}+\frac{C_{00,00}^{00,1}}{4},
$$




$$
C_{00,0000,0}^{00,0000,0, N}=-\frac{C_{00,00}^{00,0}}{8}-\frac{C_{00,00}^{00,1}}{4}+\frac{\sqrt{3}}{8} C_{00,20}^{00,0}+\frac{\sqrt{3}}{4} C_{00,20}^{00,1} .
$$

For higher-order terms of the EDF, we note that terms depending on gradients $D_{m 0}$ of nonlocal densities do contribute provided that they are coupled to a scalar. There are 1, 4, and 8 such a terms at second, fourth, and sixth order, respectively; below they are shown in bold face. For these densities, the local limit does not give rise to the cancellation of the corresponding term of the functional.

At second-order, we obtain three nonvanishing coupling constants, which are given by,

$$
\begin{aligned}
C_{00,2000,0}^{00,0000,0, L}= & \frac{1}{4} C_{00,00}^{20,0}+\frac{1}{8} C_{00,00}^{20,1}+\frac{1}{4} C_{11,00}^{11,0}+\frac{1}{8} C_{11,00}^{11,1} \\
C_{00,2000,0}^{00,0000,0, N}= & -\frac{1}{16} C_{00,00}^{20,0}-\frac{1}{8} C_{00,00}^{20,1}+\frac{1}{16} \sqrt{3} C_{00,20}^{20,0}+\frac{1}{8} \sqrt{3} C_{00,20}^{20,1} \\
& +\frac{1}{16} C_{11,00}^{11,0}+\frac{1}{8} C_{11,00}^{11,1}-\frac{1}{16} \sqrt{3} C_{11,20}^{11,0}-\frac{1}{8} \sqrt{3} C_{11,20}^{11,1} \\
C_{\mathbf{2 0 , 0 0 0 0 , 0}=}^{\mathbf{0 0 , 0 0 0 0 , 0}=} & \frac{1}{64} C_{00,00}^{20,0}+\frac{1}{32} C_{00,00}^{20,1}-\frac{1}{64} \sqrt{3} C_{00,20}^{20,0}-\frac{1}{32} \sqrt{3} C_{00,20}^{20,1} \\
& +\frac{1}{64} C_{11,00}^{11,0}+\frac{1}{32} C_{11,00}^{11,1}-\frac{1}{64} \sqrt{3} C_{11,20}^{11,0}-\frac{1}{32} \sqrt{3} C_{11,20}^{11,1} .
\end{aligned}
$$

At fourth (sixth) order, there are 8 (12) non-vanishing coupling constants, which are linear combinations of the 16 (24) parameters corresponding to the central terms of the pseudopotential. These lengthy expressions are collected in the supplemental material. Here we only list these non-vanishing coupling constants, which at fourth and sixth order are $C_{00,2000,0}^{00,2000,0, L}, C_{00,2000,0}^{00,2000,0, N}, C_{00,4000,0}^{00,0000, L}$,

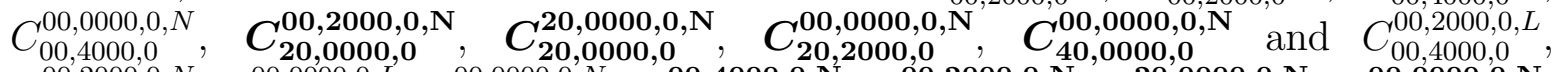

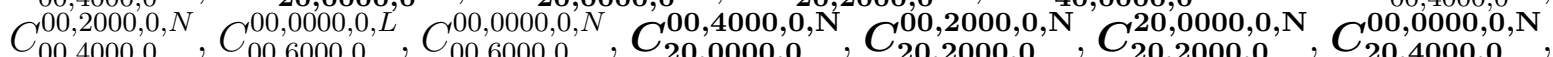
$C_{40,0000,0}^{00,2000,0, \mathrm{~N}}, C_{40,0000,0}^{20,0000,0, \mathrm{~N}}, C_{40,2000,0}^{00,0000,0, \mathrm{~N}}, C_{60,0000,0}^{00,0000,0, \mathrm{~N}}$, respectively.

\section{Cartesian representation of the central pseudopotential}

In this section, we present derivation in the Cartesian representation of the nonlocal EDF that stems from the central $(\tilde{S}=0)$ component of the regularized pseudopotential. This derivation is useful, because it establishes a clear connection of results presented in Sec. 2 with the Skyrme functional, which, in fact, constitutes the local limit of the nonlocal EDF. We used the explicit expressions for the Cartesian NLO functional, derived from the central finite-range pseudopotential, to benchmark their spherical counterparts. We checked explicitly that the two representations are equivalent when the relations between Cartesian and spherical local densities [9] and parameters of the interactions [1] were applied.

Following Refs. [3, 17, 26], we define the Cartesian form of the (non-antisymmetrized) central pseudopotential (2)-(3) in two equivalent representations as

$$
\mathcal{V}_{C}=\sum_{n j}\left(W_{j}^{(n)} \hat{1}_{\sigma} \hat{1}_{\tau}+B_{j}^{(n)} \hat{1}_{\tau} \hat{P}^{\sigma}-H_{j}^{(n)} \hat{1}_{\sigma} \hat{P}^{\tau}-M_{j}^{(n)} \hat{P}^{\sigma} \hat{P}^{\tau}\right)
$$


Nonlocal low-energy nuclear EDF

$$
\times \hat{O}_{j}^{(n)}\left(\boldsymbol{k}^{\prime}, \boldsymbol{k}\right) \delta\left(\boldsymbol{r}_{1}^{\prime}-\boldsymbol{r}_{1}\right) \delta\left(\boldsymbol{r}_{2}^{\prime}-\boldsymbol{r}_{2}\right) g_{a}\left(\boldsymbol{r}_{1}-\boldsymbol{r}_{2}\right)
$$

and

$$
\begin{array}{r}
\mathcal{V}_{C}=\sum_{n j} t_{j}^{(n)}\left(\hat{1}_{\sigma} \hat{1}_{\tau}+x_{j}^{(n)} \hat{1}_{\tau} \hat{P}^{\sigma}-y_{j}^{(n)} \hat{1}_{\sigma} \hat{P}^{\tau}-z_{j}^{(n)} \hat{P}^{\sigma} \hat{P}^{\tau}\right) \\
\times \hat{O}_{j}^{(n)}\left(\boldsymbol{k}^{\prime}, \boldsymbol{k}\right) \delta\left(\boldsymbol{r}_{1}^{\prime}-\boldsymbol{r}_{1}\right) \delta\left(\boldsymbol{r}_{2}^{\prime}-\boldsymbol{r}_{2}\right) g_{a}\left(\boldsymbol{r}_{1}-\boldsymbol{r}_{2}\right),
\end{array}
$$

which contain the standard identity $\left(\hat{1}_{\sigma, \tau}\right)$ and exchange $\left(\hat{P}^{\sigma, \tau}\right)$ operators in spin and isospin spaces. In these expressions, indexes $n$ denote the orders of differential operators $\hat{O}_{j}^{(n)}\left(\boldsymbol{k}^{\prime}, \boldsymbol{k}\right)$ and indexes $j$ number different operators of the same order. The first form, Eq. (37), generalizes the Gogny interaction by adding terms of higher orders $n>0$, whereas the second form, Eq. (38), generalizes the Skyrme interaction by adding two additional exchange terms. Obviously, the two forms are simply related one to another by the following relations between their strength parameters: $W_{j}^{(n)}=t_{j}^{(n)}$, $B_{j}^{(n)}=t_{j}^{(n)} x_{j}^{(n)}, H_{j}^{(n)}=t_{j}^{(n)} y_{j}^{(n)}$, and $M_{j}^{(n)}=t_{j}^{(n)} z_{j}^{(n)}$.

Differential operators $\hat{O}_{j}^{(n)}\left(\boldsymbol{k}^{\prime}, \boldsymbol{k}\right)$ are scalar polynomial functions of two vectors, so owing to the GCH theorem [24], they must be polynomials of three elementary scalars: $\boldsymbol{k}^{2}, \boldsymbol{k}^{\prime 2}$, and $\boldsymbol{k}^{\prime} \cdot \boldsymbol{k}$. Hermiticity of the operators $\hat{O}_{j}^{(n)}\left(\boldsymbol{k}^{\prime}, \boldsymbol{k}\right)$ can be enforced by using expressions symmetric with respect to exchanging $\boldsymbol{k}^{\prime *}$ and $\boldsymbol{k}$; therefore, it is convenient to build them from the following three scalars,

$$
\begin{aligned}
& \hat{T}_{1}=\frac{1}{2}\left(\boldsymbol{k}^{\prime * 2}+\boldsymbol{k}^{2}\right), \\
& \hat{T}_{2}=\boldsymbol{k}^{\prime *} \cdot \boldsymbol{k}, \\
& \hat{T}_{3}=\frac{1}{2}\left(\boldsymbol{k}^{\prime * 2}-\boldsymbol{k}^{2}\right),
\end{aligned}
$$

with the condition that only even powers of $\hat{T}_{3}$ can appear. In terms of $\hat{T}_{1}, \hat{T}_{2}$, and $\hat{T}_{3}$, we now can define the following differential operators:

$$
\begin{aligned}
& \hat{O}_{1}^{(0)}\left(\boldsymbol{k}^{\prime}, \boldsymbol{k}\right)=\hat{1}, \\
& \hat{O}_{1}^{(2)}\left(\boldsymbol{k}^{\prime}, \boldsymbol{k}\right)=\hat{T}_{1}, \\
& \hat{O}_{2}^{(2)}\left(\boldsymbol{k}^{\prime}, \boldsymbol{k}\right)=\hat{T}_{2}, \\
& \hat{O}_{1}^{(4)}\left(\boldsymbol{k}^{\prime}, \boldsymbol{k}\right)=\hat{T}_{1}^{2}+\hat{T}_{2}^{2}, \\
& \hat{O}_{2}^{(4)}\left(\boldsymbol{k}^{\prime}, \boldsymbol{k}\right)=2 \hat{T}_{1} \hat{T}_{2}, \\
& \hat{O}_{3}^{(4)}\left(\boldsymbol{k}^{\prime}, \boldsymbol{k}\right)=\hat{T}_{1}^{2}-\hat{T}_{2}^{2}, \\
& \hat{O}_{4}^{(4)}\left(\boldsymbol{k}^{\prime}, \boldsymbol{k}\right)=\hat{T}_{3}^{2}, \\
& \hat{O}_{1}^{(6)}\left(\boldsymbol{k}^{\prime}, \boldsymbol{k}\right)=\hat{T}_{1}^{3}+3 \hat{T}_{1} \hat{T}_{2}^{2}, \\
& \hat{O}_{2}^{(6)}\left(\boldsymbol{k}^{\prime}, \boldsymbol{k}\right)=\hat{T}_{2}^{3}+3 \hat{T}_{1}^{2} \hat{T}_{2}, \\
& \hat{O}_{3}^{(6)}\left(\boldsymbol{k}^{\prime}, \boldsymbol{k}\right)=\hat{T}_{1}^{3}-\hat{T}_{1} \hat{T}_{2}^{2}, \\
& \hat{O}_{4}^{(6)}\left(\boldsymbol{k}^{\prime}, \boldsymbol{k}\right)=\hat{T}_{2}^{3}-\hat{T}_{1}^{2} \hat{T}_{2}, \\
& \hat{O}_{5}^{(6)}\left(\boldsymbol{k}^{\prime}, \boldsymbol{k}\right)=\hat{T}_{3}^{2} \hat{T}_{1}, \\
& \hat{O}_{6}^{(6)}\left(\boldsymbol{k}^{\prime}, \boldsymbol{k}\right)=\hat{T}_{3}^{2} \hat{T}_{2} .
\end{aligned}
$$


Because for every operator $\hat{O}_{j}^{(n)}\left(\boldsymbol{k}^{\prime}, \boldsymbol{k}\right)$ there appear in Eqs. (37) and (38) four different spin-isospin terms, we recover here the numbers of central terms shown in Table 1 .

Of course, at any given order, the choice of polynomials of $\hat{T}_{1}, \hat{T}_{2}$, and $\hat{T}_{3}$ is quite arbitrary - with only requirement that these polynomials be linearly independent. Definitions (42)-(54) were chosen so as to naturally link them to the standard Skyrme interaction, for which we have

$$
\begin{array}{ll}
t_{0}=t_{1}^{(0)}, & x_{0}=x_{1}^{(0)}, \\
t_{1}=t_{1}^{(2)}, & x_{1}=x_{1}^{(2)}, \\
t_{2}=t_{2}^{(2)}, & x_{2}=x_{2}^{(2)},
\end{array}
$$

and to encompass definitions of fourth-order parameters $t_{1}^{(4)}, t_{2}^{(4)}, x_{1}^{(4)}$, and $x_{2}^{(4)}$ introduced in Ref. [26]. For a lighter notation used below, we also define the analogous parameters:

$$
\begin{array}{ll}
y_{0}=y_{1}^{(0)}, & z_{0}=z_{1}^{(0)}, \\
y_{1}=y_{1}^{(2)}, & z_{1}=z_{1}^{(2)}, \\
y_{2}=y_{2}^{(2)}, & z_{2}=z_{2}^{(2)} .
\end{array}
$$

At higher orders, we picked the $j=1$ and 2 terms so as to have at any order $n>0$,

$$
\hat{O}_{1}^{(n)}\left(\boldsymbol{k}^{\prime}, \boldsymbol{k}\right)-\hat{O}_{2}^{(n)}\left(\boldsymbol{k}^{\prime}, \boldsymbol{k}\right)=\left(\hat{T}_{1}-\hat{T}_{2}\right)^{n / 2}=\frac{1}{2^{n}}\left(\boldsymbol{k}^{\prime *}-\boldsymbol{k}\right)^{n} \equiv \frac{1}{2^{n}}\left(\boldsymbol{k}^{\prime}+\boldsymbol{k}\right)^{n} .
$$

These particular polynomials of relative momenta are special [3], because, as it turns out, the sum of relative-momentum operators $\boldsymbol{k}^{\prime}+\boldsymbol{k}$ commutes with the locality deltas, see Sec. 5 ,

In the following we give separate expressions for the functional derived from three lowest-order terms (42)-(44) of the pseudopotential, denoting them by $\left\langle V_{i}\right\rangle$ for $i=0,1$, and 2. We also separate the local and non local terms, denoting them, respectively, by $\left\langle V_{i}^{L}\right\rangle$ and $\left\langle V_{i}^{N}\right\rangle$. To have more compact expressions, we also introduced the following combinations of parameters of the regularized interaction (55) $-(60)$ :

$$
\begin{aligned}
& A_{i}^{\rho_{0}}=\frac{1}{2} t_{i}\left(1+\frac{1}{2} x_{i}-\frac{1}{2} y_{i}-\frac{1}{4} z_{i}\right), \\
& A_{i}^{\rho_{1}}=-\frac{1}{2} t_{i}\left(\frac{1}{2} y_{i}+\frac{1}{4} z_{i}\right), \\
& A_{i}^{s_{0}}=\frac{1}{2} t_{i}\left(\frac{1}{2} x_{i}-\frac{1}{4} z_{i}\right), \\
& A_{i}^{s_{1}}=-\frac{1}{8} t_{i} z_{i} \\
& B_{i}^{\rho_{0}}=-\frac{1}{2} t_{i}\left(\frac{1}{4}+\frac{1}{2} x_{i}-\frac{1}{2} y_{i}-z_{i}\right), \\
& B_{i}^{\rho_{1}}=-\frac{1}{2} t_{i}\left(\frac{1}{4}+\frac{1}{2} x_{i}\right), \\
& B_{i}^{s_{0}}=-\frac{1}{2} t_{i}\left(\frac{1}{4}-\frac{1}{2} y_{i}\right), \\
& B_{i}^{s_{1}}=-\frac{1}{8} t_{i} .
\end{aligned}
$$




\subsection{Zero-order functional}

The leading order functional, which depends on four parameters $t_{0}, x_{0}, y_{0}$, and $z_{0}$ of the regularized interaction (38), has the form

$$
\left\langle V_{0}\right\rangle=\left\langle V_{0}^{L}\right\rangle+\left\langle V_{0}^{N}\right\rangle
$$

with

$$
\begin{aligned}
\left\langle V_{0}^{L}\right\rangle= & \int \mathrm{d} \mathbf{r}_{1} \mathrm{~d} \mathbf{r}_{2} g_{a}\left(\mathbf{r}_{1}-\mathbf{r}_{2}\right)\left[A_{0}^{\rho_{0}} \rho_{0}\left(\mathbf{r}_{1}\right) \rho_{0}\left(\mathbf{r}_{2}\right)+A_{0}^{\rho_{1}} \rho_{1}\left(\mathbf{r}_{1}\right) \rho_{1}\left(\mathbf{r}_{2}\right)\right. \\
& \left.+A_{0}^{\mathbf{s}_{0}} \mathbf{s}_{0}\left(\mathbf{r}_{1}\right) \cdot \mathbf{s}_{0}\left(\mathbf{r}_{2}\right)+A_{0}^{\mathbf{s}_{1}} \mathbf{s}_{1}\left(\mathbf{r}_{1}\right) \cdot \mathbf{s}_{1}\left(\mathbf{r}_{2}\right)\right]
\end{aligned}
$$

and

$$
\begin{aligned}
\left\langle V_{0}^{N}\right\rangle= & \int \mathrm{d} \mathbf{r}_{1} \mathrm{~d} \mathbf{r}_{2} g_{a}\left(\mathbf{r}_{1}-\mathbf{r}_{2}\right)\left[B_{0}^{\rho_{0}} \rho_{0}\left(\mathbf{r}_{2}, \mathbf{r}_{1}\right) \rho_{0}\left(\mathbf{r}_{1}, \mathbf{r}_{2}\right)\right. \\
& +B_{0}^{\rho_{1}} \rho_{1}\left(\mathbf{r}_{2}, \mathbf{r}_{1}\right) \rho_{1}\left(\mathbf{r}_{1}, \mathbf{r}_{2}\right) \\
& \left.+B_{0}^{\mathbf{s}_{0}} \mathbf{s}_{0}\left(\mathbf{r}_{2}, \mathbf{r}_{1}\right) \cdot \mathbf{s}_{0}\left(\mathbf{r}_{1}, \mathbf{r}_{2}\right)+B_{0}^{\mathbf{s}_{1}} \mathbf{s}_{1}\left(\mathbf{r}_{2}, \mathbf{r}_{1}\right) \cdot \mathbf{s}_{1}\left(\mathbf{r}_{1}, \mathbf{r}_{2}\right)\right] .
\end{aligned}
$$

Densities $\rho_{0}(\boldsymbol{r}), \rho_{1}(\boldsymbol{r}), \mathbf{s}_{0}(\boldsymbol{r})$, and $\mathbf{s}_{1}(\boldsymbol{r})$, together with their nonlocal counterparts, are the standard scalar and vector densities in spin and isospin spaces, as defined in Ref. [23. By taking the zero-range limit, which amounts to bringing in Eqs. (71) and (72) the regularized delta function $g_{a}(\boldsymbol{r})$ to its Dirac delta limit, and using relations (62), one recovers the standard local form of the zero-order functional,

$$
\begin{aligned}
\left\langle V_{0}\right\rangle= & \frac{1}{2} t_{0} \int \mathrm{d} \mathbf{r}\left\{\frac{3}{4}\left(1+z_{0}\right) \rho_{0}^{2}-\left[\frac{1}{4}\left(1+z_{0}\right)+\frac{1}{2}\left(x_{0}+y_{0}\right)\right] \rho_{1}^{2}\right. \\
& \left.-\left[\frac{1}{4}\left(1+z_{0}\right)-\frac{1}{2}\left(x_{0}+y_{0}\right)\right] \mathbf{s}_{0}^{2}-\frac{1}{4}\left(1+z_{0}\right) \mathbf{s}_{1}^{2}\right\} .
\end{aligned}
$$

We explicitly verified that the Cartesian zero-order nonlocal EDF (170) is exactly equivalent to that in the spherical-tensor representation. To show this equivalence, we made use of the relations of conversions between spherical and Cartesian local densities [9], which are also valid for nonlocal densities. In the same way, one finds the relations of conversions between the parameters of the zero-order regularized pseudopotential (3) and those of its Cartesian form (38), which read

$$
\begin{aligned}
& C_{00,00}^{00,0}=t_{0}+\frac{1}{2} t_{0} x_{0}, \\
& C_{00,00}^{00,1}=-\frac{1}{2} t_{0} z_{0}-t_{0} y_{0}, \\
& C_{00,20}^{00,0}=-\frac{\sqrt{3}}{2} t_{0} x_{0}, \\
& C_{00,20}^{00,1}=\frac{\sqrt{3}}{2} t_{0} z_{0} .
\end{aligned}
$$

\subsection{Second-order functional}

At NLO, one obtains the EDF corresponding to the $i=1$ term of the regularized pseudopotential (43) by applying on all possible bilinear densities the relative-momentum 
operator:

$$
\boldsymbol{k}^{\prime * 2}+\boldsymbol{k}^{2}=-\frac{1}{4}\left(\boldsymbol{\nabla}_{1}^{\prime 2}-2 \boldsymbol{\nabla}_{1}^{\prime} \cdot \boldsymbol{\nabla}_{2}^{\prime}+\boldsymbol{\nabla}_{2}^{\prime 2}+\boldsymbol{\nabla}_{1}^{2}-2 \boldsymbol{\nabla}_{1} \cdot \boldsymbol{\nabla}_{2}+\boldsymbol{\nabla}_{2}^{2}\right)
$$

Using once again for densities the same notations as in Ref. [23], we get the quasilocal term,

$$
\begin{aligned}
\left\langle V_{1}^{L}\right\rangle= & -\frac{1}{2} \int \mathrm{d} \mathbf{r}_{1} \mathrm{~d} \mathbf{r}_{2} g_{a}\left(\mathbf{r}_{1}-\mathbf{r}_{2}\right) \\
\times & \left\{A_{1}^{\rho_{0}}\left[\left[\frac{1}{2} \Delta \rho_{0}\left(\mathbf{r}_{1}\right)-\tau_{0}\left(\mathbf{r}_{1}\right)\right] \rho_{0}\left(\mathbf{r}_{2}\right)-\frac{1}{4} \boldsymbol{\nabla} \rho_{0}\left(\mathbf{r}_{1}\right) \cdot \boldsymbol{\nabla} \rho_{0}\left(\mathbf{r}_{2}\right)+\mathbf{j}_{0}\left(\mathbf{r}_{1}\right) \cdot \mathbf{j}_{0}\left(\mathbf{r}_{2}\right)\right]\right. \\
& +A_{1}^{\rho_{1}}\left[\left[\frac{1}{2} \Delta \rho_{1}\left(\mathbf{r}_{1}\right)-\tau_{1}\left(\mathbf{r}_{1}\right)\right] \rho_{1}\left(\mathbf{r}_{2}\right)-\frac{1}{4} \boldsymbol{\nabla} \rho_{1}\left(\mathbf{r}_{1}\right) \cdot \nabla \rho_{1}\left(\mathbf{r}_{2}\right)+\mathbf{j}_{1}\left(\mathbf{r}_{1}\right) \cdot \mathbf{j}_{1}\left(\mathbf{r}_{2}\right)\right] \\
+ & A_{1}^{\mathbf{s}_{0}}\left[\left[\frac{1}{2} \Delta \mathbf{s}_{0}\left(\mathbf{r}_{1}\right)-\mathbf{T}_{0}\left(\mathbf{r}_{1}\right)\right] \cdot \mathbf{s}_{0}\left(\mathbf{r}_{2}\right)\right. \\
& \left.\quad-\frac{1}{4} \boldsymbol{\nabla} \otimes \mathbf{s}_{0}\left(\mathbf{r}_{1}\right) \cdot \boldsymbol{\nabla} \otimes \mathbf{s}_{0}\left(\mathbf{r}_{2}\right)+\mathrm{J}_{0}\left(\mathbf{r}_{1}\right) \cdot \mathrm{J}_{0}\left(\mathbf{r}_{2}\right)\right] \\
+ & A_{1}^{\mathbf{s}_{1}}\left[\left[\frac{1}{2} \Delta \mathbf{s}_{1}\left(\mathbf{r}_{1}\right)-\mathbf{T}_{1}\left(\mathbf{r}_{1}\right)\right] \cdot \mathbf{s}_{1}\left(\mathbf{r}_{2}\right)\right. \\
& \left.\left.\quad-\frac{1}{4} \boldsymbol{\nabla} \otimes \mathbf{s}_{1}\left(\mathbf{r}_{1}\right) \cdot \boldsymbol{\nabla} \otimes \mathbf{s}_{1}\left(\mathbf{r}_{2}\right)+J_{1}\left(\mathbf{r}_{1}\right) \cdot \mathrm{J}_{1}\left(\mathbf{r}_{2}\right)\right]\right\}
\end{aligned}
$$

and the nonlocal term,

$$
\begin{aligned}
\left\langle V_{1}^{N}\right\rangle= & -\frac{1}{4} \int \mathrm{d} \mathbf{r}_{1} \mathrm{~d} \mathbf{r}_{2} g_{a}\left(\mathbf{r}_{1}-\mathbf{r}_{2}\right) \\
\times & \left\{B _ { 1 } ^ { \rho _ { 0 } } \left[\rho_{0}\left(\mathbf{r}_{2}, \mathbf{r}_{1}\right) \Delta \rho_{0}\left(\mathbf{r}_{1}, \mathbf{r}_{2}\right)-2 \rho_{0}\left(\mathbf{r}_{2}, \mathbf{r}_{1}\right) \tau_{0}\left(\mathbf{r}_{1}, \mathbf{r}_{2}\right)\right.\right. \\
& \left.\quad-\frac{1}{2} \boldsymbol{\nabla} \rho_{0}\left(\mathbf{r}_{2}, \mathbf{r}_{1}\right) \cdot \boldsymbol{\nabla} \rho_{0}\left(\mathbf{r}_{1}, \mathbf{r}_{2}\right)\right] \\
& +B_{1}^{\rho_{1}}\left[\rho_{1}\left(\mathbf{r}_{2}, \mathbf{r}_{1}\right) \Delta \rho_{1}\left(\mathbf{r}_{1}, \mathbf{r}_{2}\right)-2 \rho_{1}\left(\mathbf{r}_{2}, \mathbf{r}_{1}\right) \tau_{1}\left(\mathbf{r}_{1}, \mathbf{r}_{2}\right)\right. \\
& \left.\quad-\frac{1}{2} \boldsymbol{\nabla} \rho_{1}\left(\mathbf{r}_{2}, \mathbf{r}_{1}\right) \cdot \nabla \rho_{1}\left(\mathbf{r}_{1}, \mathbf{r}_{2}\right)\right] \\
& +B_{1}^{\mathbf{s}_{0}}\left[\mathbf{s}_{0}\left(\mathbf{r}_{2}, \mathbf{r}_{1}\right) \cdot \Delta \mathbf{s}_{0}\left(\mathbf{r}_{1}, \mathbf{r}_{2}\right)-2 \mathbf{s}_{0}\left(\mathbf{r}_{2}, \mathbf{r}_{1}\right) \cdot \mathbf{T}_{0}\left(\mathbf{r}_{1}, \mathbf{r}_{2}\right)\right. \\
& \left.\quad-\frac{1}{2} \boldsymbol{\nabla} \otimes \mathbf{s}_{0}\left(\mathbf{r}_{2}, \mathbf{r}_{1}\right) \cdot \boldsymbol{\nabla} \otimes \mathbf{s}_{0}\left(\mathbf{r}_{1}, \mathbf{r}_{2}\right)+2 \mathrm{~J}_{0}\left(\mathbf{r}_{2}, \mathbf{r}_{1}\right) \cdot \mathrm{J}_{0}\left(\mathbf{r}_{1}, \mathbf{r}_{2}\right)\right] \\
& +B_{1}^{\mathbf{s}_{1}}\left[\mathbf{s}_{1}\left(\mathbf{r}_{2}, \mathbf{r}_{1}\right) \cdot \Delta \mathbf{s}_{1}\left(\mathbf{r}_{1}, \mathbf{r}_{2}\right)-2 \mathbf{s}_{1}\left(\mathbf{r}_{2}, \mathbf{r}_{1}\right) \cdot \mathbf{T}_{1}\left(\mathbf{r}_{1}, \mathbf{r}_{2}\right)\right. \\
& \left.\left.\quad-\frac{1}{2} \boldsymbol{\nabla} \otimes \mathbf{s}_{1}\left(\mathbf{r}_{2}, \mathbf{r}_{1}\right) \cdot \boldsymbol{\nabla} \otimes \mathbf{s}_{1}\left(\mathbf{r}_{1}, \mathbf{r}_{2}\right)+2 \mathrm{~J}_{1}\left(\mathbf{r}_{2}, \mathbf{r}_{1}\right) \cdot \mathrm{J}_{1}\left(\mathbf{r}_{1}, \mathbf{r}_{2}\right)\right]\right\} .
\end{aligned}
$$

In Eqs. (79) and (80), similarly as in Sec. 2, depending on the context, the nabla operator $\boldsymbol{\nabla}$ acts on the local densities as $\boldsymbol{\nabla}_{1}$ or $\boldsymbol{\nabla}_{2}$ and on the nonlocal densities as $\boldsymbol{\nabla}_{1}+\boldsymbol{\nabla}_{2}$.

By taking the local limit of functionals (79) and (80), we again recover the quasilocal Cartesian EDF, which reads

$$
\begin{aligned}
\left\langle V_{1}\right\rangle=\frac{1}{4} t_{1} \int \mathrm{d} \mathbf{r} & \frac{3}{4}\left(1+z_{1}\right)\left[\tau_{0} \rho_{0}-\mathbf{j}_{0}^{2}-\frac{3}{4} \rho_{0} \Delta \rho_{0}\right] \\
& -\left[\frac{1}{4}\left(1+z_{1}\right)+\frac{1}{2}\left(x_{1}+y_{1}\right)\right]\left[\tau_{1} \rho_{1}-\mathbf{j}_{1}^{2}-\frac{3}{4} \rho_{1} \Delta \rho_{1}\right] \\
& -\left[\frac{1}{4}\left(1+z_{1}\right)-\frac{1}{2}\left(x_{1}+y_{1}\right)\right]\left[\mathbf{T}_{0} \cdot \mathbf{s}_{0}-\mathrm{J}_{0}^{2}-\frac{3}{4} \mathbf{s}_{0} \cdot \Delta \mathbf{s}_{0}\right] \\
& -\frac{1}{4}\left(1+z_{1}\right)\left[\mathbf{T}_{1} \cdot \mathbf{s}_{1}-J_{1}^{2}-\frac{3}{4} \mathbf{s}_{1} \cdot \Delta \mathbf{s}_{1}\right] .
\end{aligned}
$$

Finally, one obtains the EDF corresponding to the $i=2$ term of the regularized pseudopotential (44) using the relative-momentum operator:

$$
\boldsymbol{k}^{\prime *} \cdot \boldsymbol{k}=\frac{1}{4}\left(\nabla_{1}^{\prime} \cdot \nabla_{1}-\nabla_{2}^{\prime} \cdot \nabla_{1}+\nabla_{2}^{\prime} \cdot \nabla_{2}-\nabla_{1}^{\prime} \cdot \nabla_{2}\right),
$$


with the result

$$
\begin{aligned}
\left\langle V_{2}^{L}\right\rangle= & \frac{1}{2} \int \mathrm{d} \mathbf{r}_{1} \mathrm{~d} \mathbf{r}_{2} g_{a}\left(\mathbf{r}_{1}-\mathbf{r}_{2}\right) \\
\times & \left\{A_{2}^{\rho_{0}}\left[\rho_{0}\left(\mathbf{r}_{1}\right) \tau_{0}\left(\mathbf{r}_{2}\right)-\frac{1}{4} \nabla \rho_{0}\left(\mathbf{r}_{1}\right) \cdot \nabla \rho_{0}\left(\mathbf{r}_{2}\right)-\mathbf{j}_{0}\left(\mathbf{r}_{1}\right) \cdot \mathbf{j}_{0}\left(\mathbf{r}_{2}\right)\right]\right. \\
& +A_{2}^{\rho_{1}}\left[\rho_{1}\left(\mathbf{r}_{1}\right) \tau_{1}\left(\mathbf{r}_{2}\right)-\frac{1}{4} \nabla \rho_{1}\left(\mathbf{r}_{1}\right) \cdot \nabla \rho_{1}\left(\mathbf{r}_{2}\right)-\mathbf{j}_{1}\left(\mathbf{r}_{1}\right) \cdot \mathbf{j}_{1}\left(\mathbf{r}_{2}\right)\right] \\
& +A_{2}^{\mathbf{s}_{0}}\left[\mathbf{s}_{0}\left(\mathbf{r}_{1}\right) \cdot \mathbf{T}_{0}\left(\mathbf{r}_{2}\right)-\frac{1}{4} \boldsymbol{\nabla} \otimes \mathbf{s}_{0}\left(\mathbf{r}_{1}\right) \cdot \boldsymbol{\nabla} \otimes \mathbf{s}_{0}\left(\mathbf{r}_{2}\right)-\mathrm{J}_{0}\left(\mathbf{r}_{1}\right) \cdot \mathrm{J}_{0}\left(\mathbf{r}_{2}\right)\right] \\
& \left.+A_{2}^{\mathbf{s}_{1}}\left[\mathbf{s}_{1}\left(\mathbf{r}_{1}\right) \cdot \mathbf{T}_{1}\left(\mathbf{r}_{2}\right)-\frac{1}{4} \boldsymbol{\nabla} \otimes \mathbf{s}_{1}\left(\mathbf{r}_{1}\right) \cdot \boldsymbol{\nabla} \otimes \mathbf{s}_{1}\left(\mathbf{r}_{2}\right)-\mathrm{J}_{1}\left(\mathbf{r}_{1}\right) \cdot \mathrm{J}_{1}\left(\mathbf{r}_{2}\right)\right]\right\}
\end{aligned}
$$

and

$$
\begin{aligned}
\left\langle V_{2}^{N}\right\rangle= & \frac{1}{2} \int \mathrm{d} \mathbf{r}_{1} \mathrm{~d} \mathbf{r}_{2} g_{a}\left(\mathbf{r}_{1}-\mathbf{r}_{2}\right) \\
\times & \left\{B_{2}^{\rho_{0}}\left[\frac{1}{4} \boldsymbol{\nabla} \rho_{0}\left(\mathbf{r}_{2}, \mathbf{r}_{1}\right) \cdot \nabla \rho_{0}\left(\mathbf{r}_{1}, \mathbf{r}_{2}\right)+\mathbf{j}_{0}\left(\mathbf{r}_{2}, \mathbf{r}_{1}\right) \cdot \mathbf{j}_{0}\left(\mathbf{r}_{1}, \mathbf{r}_{2}\right)-\rho_{0}\left(\mathbf{r}_{2}, \mathbf{r}_{1}\right) \tau_{0}\left(\mathbf{r}_{1}, \mathbf{r}_{2}\right)\right]\right. \\
+ & B_{2}^{\rho_{1}}\left[\frac{1}{4} \boldsymbol{\nabla} \rho_{1}\left(\mathbf{r}_{2}, \mathbf{r}_{1}\right) \cdot \nabla \rho_{1}\left(\mathbf{r}_{1}, \mathbf{r}_{2}\right)+\mathbf{j}_{1}\left(\mathbf{r}_{2}, \mathbf{r}_{1}\right) \cdot \mathbf{j}_{1}\left(\mathbf{r}_{1}, \mathbf{r}_{2}\right)-\rho_{1}\left(\mathbf{r}_{2}, \mathbf{r}_{1}\right) \tau_{1}\left(\mathbf{r}_{1}, \mathbf{r}_{2}\right)\right] \\
& +B_{2}^{\mathbf{s}_{0}}\left[\frac{1}{4} \boldsymbol{\nabla} \otimes \mathbf{s}_{0}\left(\mathbf{r}_{2}, \mathbf{r}_{1}\right) \cdot \boldsymbol{\nabla} \otimes \mathbf{s}_{0}\left(\mathbf{r}_{1}, \mathbf{r}_{2}\right)+\mathrm{J}_{0}\left(\mathbf{r}_{2}, \mathbf{r}_{1}\right) \cdot \mathrm{J}_{0}\left(\mathbf{r}_{1}, \mathbf{r}_{2}\right)\right. \\
& \left.\quad-\mathbf{s}_{0}\left(\mathbf{r}_{2}, \mathbf{r}_{1}\right) \cdot \mathbf{T}_{0}\left(\mathbf{r}_{1}, \mathbf{r}_{2}\right)\right] \\
& +B_{2}^{\mathbf{s}_{1}}\left[\frac{1}{4} \boldsymbol{\nabla} \otimes \mathbf{s}_{1}\left(\mathbf{r}_{2}, \mathbf{r}_{1}\right) \cdot \boldsymbol{\nabla} \otimes \mathbf{s}_{1}\left(\mathbf{r}_{1}, \mathbf{r}_{2}\right)+\mathrm{J}_{1}\left(\mathbf{r}_{2}, \mathbf{r}_{1}\right) \cdot \mathrm{J}_{1}\left(\mathbf{r}_{1}, \mathbf{r}_{2}\right)\right. \\
& \left.\left.\quad-\mathbf{s}_{1}\left(\mathbf{r}_{2}, \mathbf{r}_{1}\right) \cdot \mathbf{T}_{1}\left(\mathbf{r}_{1}, \mathbf{r}_{2}\right)\right]\right\},
\end{aligned}
$$

whereas the corresponding quasilocal Cartesian EDF reads,

$$
\begin{aligned}
\left\langle V_{2}\right\rangle=\frac{1}{4} t_{2} \int \mathrm{d} \mathbf{r}\{ & {\left[\frac{5}{4}\left(1-z_{2}\right)+x_{2}-y_{2}\right]\left[\rho_{0} \tau_{0}+\frac{1}{4} \rho_{0} \Delta \rho_{0}-\mathbf{j}_{0}^{2}\right] } \\
& +\left[\frac{1}{4}\left(1-z_{2}\right)+\frac{1}{2}\left(x_{2}-y_{2}\right)\right]\left[\rho_{1} \tau_{1}+\frac{1}{4} \rho_{1} \Delta \rho_{1}-\mathbf{j}_{1}^{2}\right] \\
& +\left[\frac{1}{4}\left(1-z_{2}\right)+\frac{1}{2}\left(x_{2}-y_{2}\right)\right]\left[\mathbf{s}_{0} \cdot \mathbf{T}_{0}+\frac{1}{4} \mathbf{s}_{0} \cdot \Delta \mathbf{s}_{0}-\mathbf{J}_{0}^{2}\right] \\
& \left.+\frac{1}{4}\left(1-z_{2}\right)\left[\mathbf{s}_{1} \cdot \mathbf{T}_{1}+\frac{1}{4} \mathbf{s}_{1} \cdot \Delta \mathbf{s}_{1}-\mathbf{J}_{1}^{2}\right]\right\} .
\end{aligned}
$$

The second-order Cartesian EDF (79, the spherical-tensor representation. The corresponding relations of conversions between the parameters of the second-order regularized pseudopotential (3) and those of its Cartesian form (38) read

$$
\begin{aligned}
& C_{00,00}^{20,0}=\sqrt{3} t_{1}+\frac{\sqrt{3}}{2} t_{1} x_{1}, \\
& C_{00,00}^{20,1}=-\sqrt{3} t_{1} y_{1}-\frac{\sqrt{3}}{2} t_{1} z_{1}, \\
& C_{00,20}^{20,0}=-\frac{3}{2} t_{1} x_{1}, \\
& C_{00,20}^{20,1}=\frac{3}{2} t_{1} z_{1}, \\
& C_{11,00}^{11,0}=\sqrt{3} t_{2}+\frac{\sqrt{3}}{2} t_{2} x_{2}, \\
& C_{11,00}^{11,1}=-\sqrt{3} t_{2} y_{2}-\frac{\sqrt{3}}{2} t_{2} z_{2},
\end{aligned}
$$




$$
\begin{aligned}
& C_{11,20}^{11,0}=-\frac{3}{2} t_{2} x_{2}, \\
& C_{11,20}^{11,1}=\frac{3}{2} t_{2} z_{2} .
\end{aligned}
$$

\section{Central pseudopotential depending on the sum of relative momenta}

In a recent paper [3], we studied the regularized finite-range interaction as an application of the ET principles to low-energy nuclear phenomena. To give an illustrative version of such effective theory, we considered a restricted form of the finite-range pseudopotential, neglecting the $\mathrm{SO}$ and tensor parts, and treating the remaining central part as depending only on the sum of relative momenta. In the spherical-tensor pseudopotential, this amounts to considering only terms of the form

$$
\begin{aligned}
\hat{V}_{v_{12} 0}^{N, \bar{t}}= & \frac{1}{2} i^{v_{12}}\left[\left[\left(K_{11}^{\prime}+K_{11}\right)^{N}\right]_{0} \hat{S}_{v_{12} 0}\right]_{0}\left(\hat{P}^{\tau}\right)^{\bar{t}} \\
& \times\left(1-\hat{P}^{M} \hat{P}^{\sigma} \hat{P}^{\tau}\right) \delta\left(\boldsymbol{r}_{1}^{\prime}-\boldsymbol{r}_{1}\right) \delta\left(\boldsymbol{r}_{2}^{\prime}-\boldsymbol{r}_{2}\right) g_{a}(r),
\end{aligned}
$$

with $N=0,2,4$, and 6 .

Dependence on the sum of relative momenta only, that is, on $\left[\left(K_{11}^{\prime}+K_{11}\right)^{N}\right]_{0}$, is a crucial feature to obtain a functional in the form of an expansion in the length scale $a$ of the regularized delta, which we used for the study of the pseudopotentials in the ET framework. Indeed, the series in $a$ is obtained by acting with the relative-momentum operators directly and only on $g_{a}(r)$, which is possible, because the sums of relative momenta do commute with the locality deltas. This fact can be explicitly demonstrated by calculating the action of $K_{11}^{\prime}+K_{11}$ on the locality deltas, that is,

$$
\begin{aligned}
& {\left[K_{11}^{\prime}+K_{11}\right] \delta\left(\boldsymbol{r}_{1}^{\prime}-\boldsymbol{r}_{1}\right) \delta\left(\boldsymbol{r}_{2}^{\prime}-\boldsymbol{r}_{2}\right)=\left[\boldsymbol{k}^{\prime}+\boldsymbol{k}\right] \delta\left(\boldsymbol{r}_{1}^{\prime}-\boldsymbol{r}_{1}\right) \delta\left(\boldsymbol{r}_{2}^{\prime}-\boldsymbol{r}_{2}\right)} \\
& =\frac{1}{2 i}\left[\boldsymbol{\nabla}_{1}^{\prime}-\nabla_{2}^{\prime}+\nabla_{1}-\nabla_{2}\right] \delta\left(\boldsymbol{r}_{1}^{\prime}-\boldsymbol{r}_{1}\right) \delta\left(\boldsymbol{r}_{2}^{\prime}-\boldsymbol{r}_{2}\right) \\
& =\frac{1}{2 i}\left[\delta^{\prime}\left(\boldsymbol{r}_{1}^{\prime}-\boldsymbol{r}_{1}\right) \delta\left(\boldsymbol{r}_{2}^{\prime}-\boldsymbol{r}_{2}\right)-\delta\left(\boldsymbol{r}_{1}^{\prime}-\boldsymbol{r}_{1}\right) \delta^{\prime}\left(\boldsymbol{r}_{2}^{\prime}-\boldsymbol{r}_{2}\right)\right. \\
& \left.\quad-\delta^{\prime}\left(\boldsymbol{r}_{1}^{\prime}-\boldsymbol{r}_{1}\right) \delta\left(\boldsymbol{r}_{2}^{\prime}-\boldsymbol{r}_{2}\right)+\delta\left(\boldsymbol{r}_{1}^{\prime}-\boldsymbol{r}_{1}\right) \delta^{\prime}\left(\boldsymbol{r}_{2}^{\prime}-\boldsymbol{r}_{2}\right)\right] \equiv 0 .
\end{aligned}
$$

As a consequence, pseudopotentials defined by (94) are strictly equivalent to ordinary local potentials given by a series of powers of Laplacians acting on the regularized delta $g_{a}(r)$ 3].

This particular restriction of the relative-momentum operators gives rise to a set of constraints on parameters of the general second-, fourth-, and sixth-order pseudopotential (2), expressed in spherical or Cartesian forms, which we list below.

At second order, corresponding to the conditions

$$
t_{2}^{(2)}=-t_{1}^{(2)}, \quad x_{2}^{(2)}=x_{1}^{(2)}, \quad y_{2}^{(2)}=y_{1}^{(2)}, \quad x_{2}^{(2)}=x_{1}^{(2)},
$$

valid for combination of the Cartesian relative momenta [3], we get the constraints,

$$
\begin{aligned}
& C_{11,00}^{11, \bar{t}}=C_{00,00}^{20, \bar{t}}, \\
& C_{11,20}^{11, \bar{t}}=C_{00,20}^{20, \bar{t}} .
\end{aligned}
$$


The analogous relations at fourth and sixth order are found by applying the binomial expansion of the term $\left[\left(K_{11}^{\prime}+K_{11}\right)^{N}\right]_{0}$ for $N=4,6$ respectively. At fourth order for $v_{12}=0,2$ and $t_{j}^{(4)}=0$ for $j>2$, we find

$$
\begin{aligned}
& C_{11, v_{12} 0}^{31, \bar{t}}=4 C_{0,0, v_{12} 0}^{40, \bar{t}}, \\
& C_{20, v_{12} 0}^{20, \bar{t}}=3 C_{00, v_{12} 0}^{40, \bar{t}}, \\
& C_{22, v_{12} 0}^{22, \bar{t}}=3 C_{00, v_{12} 0}^{40, \bar{t}},
\end{aligned}
$$

and at sixth order we have,

$$
\begin{aligned}
& C_{11, v_{12} 0}^{51, \bar{t}}=6 C_{00, v_{12} 0}^{60, \bar{t}}, \\
& C_{20, v_{12} 0}^{40, \bar{t}}=15 C_{00, v_{12}}^{60, \bar{t}}, \\
& C_{22, v_{12} 0}^{42, \bar{t}}=15 C_{00, v_{12} 0}^{60, \bar{t}}, \\
& C_{31, v_{12} 0}^{31, \bar{t}}=10 C_{00, v_{12} 0}^{60, \bar{t}}, \\
& C_{33, v_{12} 0}^{33, \bar{t}}=10 C_{00, v_{12} 0}^{60, \bar{t}},
\end{aligned}
$$

whereas all the other parameters $C_{\tilde{n} \tilde{L}, v_{12} S}^{\tilde{n}^{\prime} \tilde{L}^{\prime}, \bar{t}}$ with $S \neq 0$ are set to zero.

\section{Cartesian representation of the $\mathrm{SO}$ and tensor pseudopotentials}

In this Section, we present construction of the SO $(\tilde{S}=1)$ and tensor $(\tilde{S}=2)$ components of the regularized pseudopotential. Following the same methodology as that introduced for central terms in Sec. [5, we define the Cartesian forms of the (non-antisymmetrized) $\mathrm{SO}$ and tensor pseudopotential, respectively, as

$$
\begin{aligned}
\mathcal{V}_{S O}=\sum_{n j} p_{j}^{(n)} & \left(\hat{1}_{\sigma} \hat{1}_{\tau}-w_{j}^{(n)} \hat{1}_{\sigma} \hat{P}^{\tau}\right) \\
& \times \hat{P}_{j}^{(n)}\left(\boldsymbol{k}^{\prime}, \boldsymbol{k}\right) \delta\left(\boldsymbol{r}_{1}^{\prime}-\boldsymbol{r}_{1}\right) \delta\left(\boldsymbol{r}_{2}^{\prime}-\boldsymbol{r}_{2}\right) g_{a}\left(\boldsymbol{r}_{1}-\boldsymbol{r}_{2}\right)
\end{aligned}
$$

and

$$
\begin{aligned}
\mathcal{V}_{T}=\sum_{n j} q_{j}^{(n)} & \left(\hat{1}_{\sigma} \hat{1}_{\tau}-v_{j}^{(n)} \hat{1}_{\sigma} \hat{P}^{\tau}\right) \\
\times & \hat{Q}_{j}^{(n)}\left(\boldsymbol{k}^{\prime}, \boldsymbol{k}\right) \delta\left(\boldsymbol{r}_{1}^{\prime}-\boldsymbol{r}_{1}\right) \delta\left(\boldsymbol{r}_{2}^{\prime}-\boldsymbol{r}_{2}\right) g_{a}\left(\boldsymbol{r}_{1}-\boldsymbol{r}_{2}\right) .
\end{aligned}
$$

The spin-dependent differential operators $\hat{P}_{j}^{(n)}\left(\boldsymbol{k}^{\prime}, \boldsymbol{k}\right)$ and $\hat{Q}_{j}^{(n)}\left(\boldsymbol{k}^{\prime}, \boldsymbol{k}\right)$ are built in the following way. First of all, they must be scalar, hermitian, and time-even operators that are obtained by coupling the space part to standard spin-vector and spin-tensor operators,

$$
\begin{aligned}
& \hat{S}_{m}=\sigma_{m}^{(1)}+\sigma_{m}^{(2)}, \\
& \hat{\mathrm{S}}_{m n}=\frac{3}{2}\left(\sigma_{m}^{(1)} \sigma_{n}^{(2)}+\sigma_{n}^{(1)} \sigma_{m}^{(2)}\right)-\delta_{m n} \boldsymbol{\sigma}^{(1)} \cdot \boldsymbol{\sigma}^{(2)},
\end{aligned}
$$

respectively, where indices $m$ and $n$ denote Cartesian components of the Pauli spin matrices. At this point we note that $\hat{\boldsymbol{S}} \hat{P}^{\sigma} \equiv \hat{\boldsymbol{S}}$ and $\hat{\mathbf{S}} \hat{P}^{\sigma} \equiv \hat{\mathrm{S}}$; therefore, the spinexchange operators $\hat{P}^{\sigma}$ do not give independent terms and thus do not appear in Eqs. (107) and (108). 
Because spin-vector operator $\hat{S}_{m}$ is even-parity and time-odd, we must build from relative-momentum operators an elementary even-parity and time-odd vector, which is only one,

$$
i\left(\boldsymbol{k}^{*} \times \boldsymbol{k}\right)_{m}=i \sum_{n l} \varepsilon_{m n l} k_{n}^{* *} k_{l} .
$$

Similarly, because spin-tensor operator $\hat{\mathrm{S}}_{m n}$ is even-parity and time-even, we must built from relative-momentum operators all elementary even-parity and time-even traceless tensors, which are three,

$$
\begin{aligned}
& (\boldsymbol{k} \otimes \boldsymbol{k})_{m n}=k_{m} k_{n}-\frac{1}{3} \delta_{m n} \boldsymbol{k} \cdot \boldsymbol{k}, \\
& \left(\boldsymbol{k}^{\prime *} \otimes \boldsymbol{k}\right)_{m n}=k_{m}^{\prime *} k_{n}-\frac{1}{3} \delta_{m n} \boldsymbol{k}^{\prime *} \cdot \boldsymbol{k}, \\
& \left(\boldsymbol{k}^{\prime *} \otimes \boldsymbol{k}^{\prime *}\right)_{m n}=k_{m}^{\prime *} k_{n}^{\prime *}-\frac{1}{3} \delta_{m n} \boldsymbol{k}^{\prime *} \cdot \boldsymbol{k}^{\prime *} .
\end{aligned}
$$

Contracting spin-vector and space-vector, as well as spin-tensor and space-tensor operators, we now obtain all suitable elementary scalar operators. Selecting convenient combinations of tensor terms, cf. Eqs. (39)-(41), we define them as,

$$
\begin{aligned}
& \hat{T}_{s}=i\left(\boldsymbol{k}^{* *} \times \boldsymbol{k}\right) \cdot\left(\boldsymbol{\sigma}^{(1)}+\boldsymbol{\sigma}^{(2)}\right), \\
& \hat{T}_{e}=\frac{1}{2}\left(\boldsymbol{k} \cdot \hat{\mathrm{S}} \cdot \boldsymbol{k}+\boldsymbol{k}^{\prime *} \cdot \hat{\mathrm{S}} \cdot \boldsymbol{k}^{\prime *}\right), \\
& \hat{T}_{o}=\boldsymbol{k}^{\prime *} \cdot \hat{\mathrm{S}} \cdot \boldsymbol{k}, \\
& \hat{T}_{a}=\frac{1}{2}\left(\boldsymbol{k} \cdot \hat{\mathrm{S}} \cdot \boldsymbol{k}-\boldsymbol{k}^{\prime *} \cdot \hat{\mathrm{S}} \cdot \boldsymbol{k}^{\prime *}\right) .
\end{aligned}
$$

Of course, at second order we have the standard SO (115), tensor-even (116), and tensorodd (117) elementary operators, see, e.g., Ref. [23], whereas the tensor-antihermitian operator (118) does not appear. Owing to the GCH theorem [24], we can now build all higher-order operators by multiplying elementary operators (115)-(118) and scalars (39)-(41). The hermiticity is then enforced by always using even numbers of antihermitian factors.

Finally, up to $\mathrm{N}^{3} \mathrm{LO}$, we obtain all possible $\mathrm{SO}$ terms,

$$
\begin{aligned}
& \hat{P}_{1}^{(2)}\left(\boldsymbol{k}^{\prime}, \boldsymbol{k}\right)=\hat{T}_{s}, \\
& \hat{P}_{1}^{(4)}\left(\boldsymbol{k}^{\prime}, \boldsymbol{k}\right)=\hat{T}_{1} \hat{T}_{s}, \\
& \hat{P}_{2}^{(4)}\left(\boldsymbol{k}^{\prime}, \boldsymbol{k}\right)=\hat{T}_{2} \hat{T}_{s}, \\
& \hat{P}_{1}^{(6)}\left(\boldsymbol{k}^{\prime}, \boldsymbol{k}\right)=\left(\hat{T}_{1}^{2}+\hat{T}_{2}^{2}\right) \hat{T}_{s}, \\
& \hat{P}_{2}^{(6)}\left(\boldsymbol{k}^{\prime}, \boldsymbol{k}\right)=2 \hat{T}_{1} \hat{T}_{2} \hat{T}_{s}, \\
& \hat{P}_{3}^{(6)}\left(\boldsymbol{k}^{\prime}, \boldsymbol{k}\right)=\left(\hat{T}_{1}^{2}-\hat{T}_{2}^{2}\right) \hat{T}_{s}, \\
& \hat{P}_{4}^{(6)}\left(\boldsymbol{k}^{\prime}, \boldsymbol{k}\right)=\hat{T}_{3}^{2} \hat{T}_{s},
\end{aligned}
$$

and all possible tensor terms,

$$
\begin{aligned}
& \hat{Q}_{1}^{(2)}\left(\boldsymbol{k}^{\prime}, \boldsymbol{k}\right)=\hat{T}_{e}, \\
& \hat{Q}_{2}^{(2)}\left(\boldsymbol{k}^{\prime}, \boldsymbol{k}\right)=\hat{T}_{o}, \\
& \hat{Q}_{1}^{(4)}\left(\boldsymbol{k}^{\prime}, \boldsymbol{k}\right)=\hat{T}_{1}\left(\hat{T}_{e}-\hat{T}_{o}\right), \\
& \hat{Q}_{2}^{(4)}\left(\boldsymbol{k}^{\prime}, \boldsymbol{k}\right)=\hat{T}_{2}\left(\hat{T}_{e}-\hat{T}_{o}\right),
\end{aligned}
$$




$$
\begin{aligned}
& \hat{Q}_{3}^{(4)}\left(\boldsymbol{k}^{\prime}, \boldsymbol{k}\right)=\hat{T}_{1}\left(\hat{T}_{e}+\hat{T}_{o}\right), \\
& \hat{Q}_{4}^{(4)}\left(\boldsymbol{k}^{\prime}, \boldsymbol{k}\right)=\hat{T}_{2}\left(\hat{T}_{e}+\hat{T}_{o}\right), \\
& \hat{Q}_{5}^{(4)}\left(\boldsymbol{k}^{\prime}, \boldsymbol{k}\right)=\hat{T}_{3} \hat{T}_{a}, \\
& \hat{Q}_{1}^{(6)}\left(\boldsymbol{k}^{\prime}, \boldsymbol{k}\right)=\left(\hat{T}_{1}^{2}+\hat{T}_{2}^{2}\right)\left(\hat{T}_{e}-\hat{T}_{o}\right), \\
& \hat{Q}_{2}^{(6)}\left(\boldsymbol{k}^{\prime}, \boldsymbol{k}\right)=2 \hat{T}_{1} \hat{T}_{2}\left(\hat{T}_{e}-\hat{T}_{o}\right), \\
& \hat{Q}_{3}^{(6)}\left(\boldsymbol{k}^{\prime}, \boldsymbol{k}\right)=\left(\hat{T}_{1}^{2}-\hat{T}_{2}^{2}\right)\left(\hat{T}_{e}-\hat{T}_{o}\right), \\
& \hat{Q}_{4}^{(6)}\left(\boldsymbol{k}^{\prime}, \boldsymbol{k}\right)=\hat{T}_{3}^{2}\left(\hat{T}_{e}-\hat{T}_{o}\right), \\
& \hat{Q}_{5}^{(6)}\left(\boldsymbol{k}^{\prime}, \boldsymbol{k}\right)=\left(\hat{T}_{1}^{2}+\hat{T}_{2}^{2}\right)\left(\hat{T}_{e}+\hat{T}_{o}\right), \\
& \hat{Q}_{6}^{(6)}\left(\boldsymbol{k}^{\prime}, \boldsymbol{k}\right)=2 \hat{T}_{1} \hat{T}_{2}\left(\hat{T}_{e}+\hat{T}_{o}\right), \\
& \hat{Q}_{7}^{(6)}\left(\boldsymbol{k}^{\prime}, \boldsymbol{k}\right)=\left(\hat{T}_{1}^{2}-\hat{T}_{2}^{2}\right)\left(\hat{T}_{e}+\hat{T}_{o}\right), \\
& \hat{Q}_{8}^{(6)}\left(\boldsymbol{k}^{\prime}, \boldsymbol{k}\right)=\hat{T}_{3}^{2}\left(\hat{T}_{e}+\hat{T}_{o}\right), \\
& \hat{Q}_{9}^{(6)}\left(\boldsymbol{k}^{\prime}, \boldsymbol{k}\right)=\hat{T}_{1} \hat{T}_{3} \hat{T}_{a}, \\
& \hat{Q}_{10}^{(6)}\left(\boldsymbol{k}^{\prime}, \boldsymbol{k}\right)=\hat{T}_{2} \hat{T}_{3} \hat{T}_{a} .
\end{aligned}
$$

We note that we here recover the numbers of SO and tensor terms given in Table 1 . We also note that the difference of the tensor-even and tensor-odd elementary operators depends only on the sum of relative momenta, that is,

$$
\hat{T}_{e}-\hat{T}_{o}=\frac{1}{2}\left(\boldsymbol{k}^{\prime}+\boldsymbol{k}\right) \cdot \hat{\mathrm{S}} \cdot\left(\boldsymbol{k}^{\prime}+\boldsymbol{k}\right) .
$$

This has motivated our choice of the $j=1$ and $j=2$ tensor terms, for which at $n>0$ we have,

$$
\hat{Q}_{1}^{(n)}\left(\boldsymbol{k}^{\prime}, \boldsymbol{k}\right)-\hat{Q}_{2}^{(n)}\left(\boldsymbol{k}^{\prime}, \boldsymbol{k}\right) \equiv \frac{1}{2^{n}}\left(\boldsymbol{k}^{\prime}+\boldsymbol{k}\right)^{n-2}\left[\left(\boldsymbol{k}^{\prime}+\boldsymbol{k}\right) \cdot \hat{\mathrm{S}} \cdot\left(\boldsymbol{k}^{\prime}+\boldsymbol{k}\right)\right] .
$$

Therefore, these particular combinations of terms commute with the locality deltas, see Sec. 5, and thus are equivalent to local tensor potentials. On the other hand, none of the SO terms has such a property.

The tensor interaction presented here may be compared with the one discussed in a recent work by Davesne et al. [27], which extends to $\mathrm{N}^{3} \mathrm{LO}$ the zero-range Cartesian pseudopotential. First we note that the $\hat{T}_{e}$ and $\hat{T}_{o}$ operators defined in the aforementioned article differ from those in Eqs. (116) and (117) by factors of 2. When $a \rightarrow 0$, one has the following correspondence between the coupling constants $q_{j}^{(n)}$ appearing in Eq. (108) and those used in Ref. [27], denoted by $t_{e}^{(n)}$ and $t_{o}^{(n)}$. (The coupling constants $w_{j}^{(n)}$ can be disregarded, because at the zero-range limit, the action of operator $\hat{P}^{\tau}$ reduces to a phase.) At second order, one recovers the pseudopotential from Ref. [27] with

$$
\begin{aligned}
& q_{1}^{(2)}=t_{e}^{(2)}, \\
& q_{2}^{(2)}=t_{o}^{(2)},
\end{aligned}
$$

at fourth order with

$$
q_{1}^{(4)}=2\left(t_{e}^{(4)}-t_{o}^{(4)}\right),
$$




$$
\begin{aligned}
& q_{2}^{(4)}=2\left(t_{o}^{(4)}-t_{e}^{(4)}\right), \\
& q_{3}^{(4)}=2\left(t_{e}^{(4)}+t_{o}^{(4)}\right), \\
& q_{4}^{(4)}=2\left(t_{e}^{(4)}+t_{o}^{(4)}\right), \\
& q_{5}^{(4)}=0,
\end{aligned}
$$

and at sixth order with

$$
\begin{aligned}
& q_{1}^{(6)}=t_{e}^{(6)}-t_{o}^{(6)}, \\
& q_{2}^{(6)}=t_{o}^{(6)}-t_{e}^{(6)}, \\
& q_{5}^{(6)}=t_{e}^{(6)}+t_{o}^{(6)}, \\
& q_{6}^{(6)}=t_{e}^{(6)}+t_{o}^{(6)}
\end{aligned}
$$

and $q_{3}^{(6)}=q_{4}^{(6)}=q_{7}^{(6)}=q_{8}^{(6)}=q_{9}^{(6)}=q_{10}^{(6)}=0$.

\section{Conclusions}

In summary, in this work, for the first time we constructed the finite-range, higher-order in derivatives pseudopotential, for which we built all terms up to sixth order $\mathrm{N}^{3} \mathrm{LO}$, and then we derived the corresponding nonlocal nuclear $\mathrm{N}^{3} \mathrm{LO}$ EDF by calculating the HF average energy over a nuclear Slater determinant. The proposed pseudopotential can thus be regarded as a generator of the EDF, which has several advantages for calculations beyond the simple HF level. First, the use of a regulator introduces a natural cut-off at high momenta, preventing divergences of pairing energies or correlation energies in the (Q)RPA limit. Then, for multi-reference EDF calculations, the link with a pseudopotential guaranties the absence of poles in energy kernels. Moreover, the fact that the pseudopotential does not depend on density guaranties that average energies are not polluted by self-interaction or self-pairing.

The ability of this pseudopotential to generate attractive pairing has not been checked yet - this is of course of crucial importance for future developments. However, the fact that at NLO it has all the features of the Gogny interaction gives us hope that this will indeed be the case. Let us also mention that all the central terms of the pseudopotential and the corresponding mean fields have already been implemented in the solver HFODD [20, 21, 22] and numerical studies of finite nuclei are under way. Moreover, a preliminary study has demonstrated that the NLO functional can provide binding energy of doubly-magic nuclei with a reasonable accuracy [17].

This work has been supported in part by the Academy of Finland and University of Jyväskylä within the FIDIPRO programme and by the Polish National Science Center under Contract No. 2012/07/B/ST2/03907. The research of FR is supported by the Natural Science and Engineering Research Council (NSERC).

[1] E. Epelbaum, H.-W. Hammer, and Ulf-G. Meißner, Rev. Mod. Phys. 81, 1773 (2009).

[2] R. Machleidt and D.-R. Entem, Phys. Rep. 503, 1 (2011).

[3] J. Dobaczewski, K. Bennaceur, and F. Raimondi, J. Phys. G 39, 125103 (2012). 
[4] D.M. Brink and E. Boeker, Nucl. Phys. A91 (1967) 1.

[5] D. Gogny, Nucl. Phys. A237, 399 (1975).

[6] D. Gogny, Proceedings of the International Conference on Nuclear Selfconsistent Fields, Trieste 1975. G. Ripka and M. Porneuf. Eds North Holland. Amsterdam, 176, 209, 265, 266 (1975).

[7] T.H.R. Skyrme, Phil. Mag. 1, 1043 (1956).

[8] T.H.R. Skyrme, Nucl. Phys. 9, 615 (1959).

[9] B.G. Carlsson, J. Dobaczewski, and M. Kortelainen, Phys. Rev. C 78, 044326 (2008).

[10] A. Gezerlis, and G. F. Bertsch, Phys. Rev. Lett. 105, 212501 (2010).

[11] F. Raimondi, B.G. Carlsson, and J. Dobaczewski, Phys. Rev. C 83, 054311 (2011).

[12] F. Raimondi, B.G. Carlsson, J. Dobaczewski, and J. Toivanen, Phys. Rev. C 84, 064303 (2011).

[13] J. Sadoudi, T. Duguet, J. Meyer, and M. Bender, Phys. Rev. C 88, 064326 (2013).

[14] M. Kortelainen, T. Lesinski, J. Moré, W. Nazarewicz, J. Sarich, N. Schunck, M.V. Stoitsov, and S. Wild, Phys. Rev. C 82, 024313 (2010).

[15] M. Kortelainen, J. McDonnell, W. Nazarewicz, P.-G. Reinhard, J. Sarich, N. Schunck, M.V. Stoitsov, and S.M. Wild, Phys. Rev. C 85, 024304 (2012).

[16] K. Washiyama, K. Bennaceur, B. Avez, M. Bender, P.-H. Heenen, V. Hellemans, Phys. Rev. C 86, 054309 (2012).

[17] K. Bennaceur, J. Dobaczewski, and F. Raimondi, arXiv:1305.7210.

[18] J. Dobaczewski, M.V. Stoitsov, W. Nazarewicz, and P.-G. Reinhard, Phys. Rev. C 76, 054315 (2007).

[19] T. Duguet, M. Bender, K. Bennaceur, D. Lacroix, and T. Lesinski, Phys. Rev. C 79, 044320 (2009).

[20] N. Schunck, J. Dobaczewski, J. McDonnell, W. Satuła, J.A. Sheikh, A. Staszczak, M. Stoitsov, P. Toivanen, Comput. Phys. Commun. 183, 166 (2012).

[21] N. Schunck et al., unpublished.

[22] J. Dobaczewski et al., HFODD home page: http://www. fuw.edu.pl/ dobaczew/hfodd/.

[23] E. Perlińska, S.G. Rohoziński, J. Dobaczewski, and W. Nazarewicz, Phys. Rev. C 69, 014316 (2004).

[24] S.G. Rohoziński, J. Dobaczewski, and W. Nazarewicz, Phys. Rev. C 81, 014313 (2010).

[25] B.G. Carlsson, J. Dobaczewski, J. Toivanen, and P. Veselý, Comput. Phys. Commun. 181, 1641 (2010).

[26] D. Davesne, A. Pastore, and J. Navarro, J. Phys. G 40, 095104 (2013).

[27] D. Davesne, A. Pastore, and J. Navarro, arXiv:1401.7914, 